\title{
A comparison of the nonlinear frictional characteristics of two-dimensional and three-dimensional models of a shallow tidal embayment
}

\author{
R.R. Grenier Jr. \\ Department of Civil Engineering, North Carolina State University, Raleigh
}

R.A. Luettich Jr.

Institute of Marine Sciences, University of North Carolina at Chapel Hill, Morehead City

\section{J.J. Westerink}

Department of Civil Engineering and Geological Sciences, University of Notre Dame, Notre Dame, Indiana

\begin{abstract}
The nonlinear frictional behavior of two-dimensional (2-D) and three-dimensional (3-D) models are compared in this study of tides in the Bight of Abaco. The shallow depths and the existence of an extensive set of tidal elevation data (five astronomical and two overtide constituents at 25 stations) from Filloux and Snyder (1979) offer an excellent opportunity to compare the effects of different frictional formulations. In addition, previous modeling efforts in the bight have consistently overpredicted the $M_{6}$ and generally overdamped the $O_{1}, K_{1}$, and $S_{2}$ tides. The results indicate that although the 2-D and 3-D models may be calibrated to produce nearly identical responses for the dominant $M_{2}$ tide, there are systematic differences in the responses of the primary overtides. These differences are explained using analytical expansions of the friction terms and are shown to be due to differences in the terms that are nonlinear in velocity and in water level. The investigation concludes that the overgeneration of $M_{6}$ and the overdamping of secondary astronomical tides will occur in 3-D models as well as 2-D models. Although several causes for these problems were considered, improvement in these constituents could be achieved only by modifying the standard quadratic friction or flow-dependent eddy viscosity relations to reduce the nonlinear frictional effect relative to the linear frictional effect. The required modifications suggest the presence of a constant background velocity, residual turbulence field, or possibly the need for a more advanced frictional closure.
\end{abstract}

\section{Introduction}

The role of friction in modeling the tidal dynamics of shallow seas and coastal regions is well appreciated and relatively well studied (at least for vertically integrated models). However, despite considerable theoretical, experimental, and numerical research, the representation and parameterization of frictional processes remains a difficult aspect of modeling. Indeed, adjustment of friction parameter(s) remains the primary means of calibration for most hydrodynamic models.

In coastal waters, tides are generally classified as astronomical tides, compound tides, or overtides. Astronomical tides result from the gravitational forces exerted by the Sun and the moon. Compound tides and overtides arise from the nonlinear interactions between constituents; they are often called shallow water tides because nonlinear phenomena (e.g., bottom friction) generally become important in shallow regions. Compound tides result from interactions between two or more constituents of different

Copyright 1995 by the American Geophysical Union.

Paper number 95JC00841.

0148-0227/95/95JC-00841\$05.00 frequency, whereas overtides result from interactions of a single constituent with itself or its overtides.

The origin and numerical simulation of shallow water tides has been the focus of much research in recent years (see LeProvost [1991] for an excellent review). Most of this work has been done using two-dimensional (2-D) vertically integrated governing equations that parameterize friction at the bottom as a quadratic function of the depth-averaged velocity. Particular emphasis has been placed on understanding the influence of the quadratic friction parameterization in rivers [Godin, 1991; Parker, 1991] and shallow seas [Snyder et al., 1979; Pingree, 1983; LeProvost and Fornerino, 1985; Speer and Aubrey, 1985; Walters, 1987; Pingree and Griffiths, 1987; Westerink et al., 1989; Bowers et al., 1991].

More recently, several three-dimensional (3-D) tidal models have appeared [e.g., Walters, 1992; Davies, 1993a; Sucsy et al., 1993; Aldridge and Davies, 1993; Davies and Aldridge, 1993; Lynch and Naimie, 1993]. In these models, frictional processes occur in the water column as well as at the bottom. Friction in the water column is often described by a flow-dependent eddy viscosity [e.g., Davies and Aldridge, 1993], while friction at the bottom is computed using either a no-slip condition or a slip condition. 
In the present study we examine the effects of friction on the tides in the Bight of Abaco, a shallow tidal embayment located in the northernmost part of the Bahama Islands. The shallow depths and varied topography of the region coupled with the existence of an extensive set of tidal elevation observations (25 stations with data available for the $\mathrm{K}_{1}, \mathrm{O}_{1}$, $\mathrm{N}_{2}, \mathrm{M}_{2}, \mathrm{~S}_{2}, \mathrm{M}_{4}$, and $\mathrm{M}_{6}$ constituents) offer an interesting challenge for a nonlinear tidal model.

Two-dimensional numerical tidal computations for the Bight of Abaco were first carried out by Sidjabat [1970] and later extended by Snyder et al., [1979] (hereinafter referred to as S79) and Westerink et al. [1989], (hereinafter referred to as W89). Although the S79 and W89 models could be tuned to successfully reproduce $M_{2}$ elevation amplitudes and phases, they were less satisfactory for the overtides and some of the secondary astronomical constituents. In particular, the $M_{6}$ amplitudes were consistently overpredicted, while the $O_{1}, K_{1}$, and $S_{2}$ tides were generally underpredicted in the interior sections of the bight.

The generation of the $M_{6}$ overtide from the $M_{2}$ constituent by the quadratic friction term in a 2-D model is one of the classical results from early analytical studies of tidal dynamics [Proudman, 1953]. In the typical case of a dominant $M_{2}$ constituent the $M_{6}$ response is nearly always too high in cases where the $M_{2}$ is modeled separately from other astronomical constituents [Pingree, 1983; LeProvost and Fornerino, 1985; LeProvost, 1991; Parker, 1991]. The $\mathrm{M}_{6}$ response is generally reduced when additional astronomical constituents are included in the model. One reason is that the friction coefficient in the $\mathrm{M}_{2}$-only simulations must compensate for the absence of other friction-producing constituents [Parker, 1991] and is therefore too high, creating too strong a source of $M_{6}$ overtide. A second reason is that compound tide interactions contribute to the reduction in the $\mathrm{M}_{6}$ overtide. For example, $M_{2}-N_{2}$ and $M_{2}-S_{2}$ interactions, which produce the $2 \mathrm{MN}_{2}$ and $2 \mathrm{MS}_{2}$ semidiumal tides, will combine with the $M_{2}$ to create $M_{6}$ contributions that are $180^{\circ}$ out of phase with the primary $M_{6}$ contributions of the $M_{2}$ constituent. LeProvost and Fornerino [1985] showed a significant reduction in $M_{6}$ in the English Channel when these secondary waves were included in their model. In the Bight of Abaco, W89 found that the addition of the $2 \mathrm{MN}_{2}$ and $2 \mathrm{MS}_{2}$ constituents significantly reduced the amplitude of the $M_{6}$ overtide. However, even after accounting for these compound tide interactions, the $M_{6}$ constituent remained overpredicted by about $50 \%$.

The overdamped secondary astronomical tides encountered by 579 and W89 are also consistent with previous twodimensional model results. The overdamping has been noted to affect semidiurnal tides as well as diumal tides [LeProvost and Fornerino, 1985; LeProvost, 1991; Bowers et al., 1991].

In summary, considerable progress has been made in modeling tides in shallow water systems in general and the Bight of Abaco in particular. Despite this progress, however, discrepancies remain between observational data and the response of (two-dimensional) tidal models in the Bight of Abaco. These discrepancies appear to be common to other tidal models and related to the representation of friction in the models.

In the present study we examine the frictional formulations of a two-dimensional and a three-dimensional tidal model. The primary difference between the models is that in the two- dimensional case, bottom stress is the only source of friction, whereas in the three-dimensional case, friction occurs in the water column and at the bottom. The responses of the models are compared in the Bight of Abaco, with particular focus on the $M_{2}$ overtides. Following this, approximate expansions for the friction terms are presented for both models and used to interpret the model responses. These expansions lead to a final set of runs which demonstrate the need to include both linear and nonlinear frictional effects in the Bight of Abaco.

While several previous investigations have used $M_{2}$ elevations and currents to examine differences between 2-D and 3-D models [e.g., Sucsy et al., 1993] and 3-D models with different eddy viscosity formulations [e.g., Davies, 1993a, Davies and Aldridge, 1993], this study uses the $M_{2}$ overtides and the secondary astronomical tides as a means of comparing models. Our investigation seeks to determine whether a 3-D model offers any advantage in an area where 2-D models have consistently failed.

\section{Model Description}

This study was conducted using the circulation model ADCIRC which includes options for either two-dimensional, depth-integrated (2DDI) or three-dimensional (3-D) solutions. A summary of key model features is provided below; considerably more detailed presentations of the model are given by Luettich et al. [1992, 1994], Kolar et al. [1994], and Westerink et al. [1994].

ADCIRC-2DDI solves the fully nonlinear shallow water equations in either spherical or Cartesian coordinate systems. Owing to the limited size of the Bight of Abaco, we use the Cartesian form of the equations

$$
\begin{gathered}
\frac{\partial \zeta}{\partial t}+\frac{\partial}{\partial x}(H U)+\frac{\partial}{\partial y}(H V)=0 \\
\frac{\partial U}{\partial t}+U \frac{\partial U}{\partial x}+V \frac{\partial U}{\partial y}-f V \\
=-\frac{\partial}{\partial x}\left[\frac{P_{a}}{\rho_{0}}+g(\zeta-\alpha \eta)\right]+\frac{1}{H}\left[M_{x}+D_{x}+\frac{\tau_{s x}}{\rho_{0}}-\frac{\tau_{b x}}{\rho_{0}}\right] \\
\frac{\partial V}{\partial t}+U \frac{\partial V}{\partial x}+V \frac{\partial V}{\partial y}+f U \\
=-\frac{\partial}{\partial y}\left[\frac{P_{a}}{\rho_{0}}+g(\zeta-\alpha \eta)\right]+\frac{1}{H}\left[M_{y}+D_{y}+\frac{\tau_{s y}}{\rho_{0}}-\frac{\tau_{b y}}{\rho_{0}}\right]
\end{gathered}
$$

where

$\zeta$ surface elevation relative to the undisturbed water level;

$U, V$ depth-averaged $x$ and $y$ velocities;

$H \quad$ total depth $(H \equiv h+\zeta)$;

$h$ bathymetric depth;

$f$ Coriolis parameter;

$\tau_{s x}, \tau_{s y} \quad$ surface stresses in the $x$ and $y$ directions;

$\tau_{b x}, \tau_{b y} \quad$ bottom stresses in the $x$ and $y$ directions;

$M_{x}, M_{y}$ horizontal turbulent momentum diffusion; 


$\begin{array}{cl}D_{x}, D_{y} & \begin{array}{l}\text { horizontal momentum dispersion } \\ \text { (resulting from vertically integrating } \\ \text { the advective terms); }\end{array} \\ \eta & \begin{array}{l}\text { Newtonian equilibrium tide potential; } \\ \text { effective Earth elasticity factor; }\end{array} \\ \rho_{0} & \text { reference density of water. }\end{array}$

For the present study the atmospheric pressure gradients, tidal potential terms, surface stresses, and horizontal momentum diffusion terms are assumed to be zero. ADCIRC2DDI also neglects the momentum dispersion terms and relates the bottom stress to the depth-averaged velocity using a quadratic friction relation:

$$
\begin{aligned}
& \frac{\tau_{b x}}{\rho_{0}}=C_{f 2 \mathrm{~d}}\left(U^{2}+V^{2}\right)^{1 / 2} U \\
& \frac{\tau_{b y}}{\rho_{\mathrm{o}}}=C_{f 2 \mathrm{~d}}\left(U^{2}+V^{2}\right)^{1 / 2} V
\end{aligned}
$$

where $C_{f 2 d}$ is the two-dimensional friction coefficient.

Prior to being discretized, the continuity and momentum equations are combined into a generalized wave continuity equation (GWCE) which has been shown to have superior numerical properties to a primitive continuity equation when a finite element method is used in space [Lynch and Gray, 1979; Kinnmark, 1985]. The GWCE is solved together with the primitive momentum equations using a Galerkin finite element method on linear triangles in space and a finite difference method in time.

ADCIRC-3D employs a mode-splitting technique to separate the horizontal and vertical problems. The solution for the surface elevation is obtained from the GWCE and constitutes the "external mode," while the solution for the vertical profile of velocity constitutes the "internal mode." Many problems of practical interest involve boundary layer flows that are characterized by velocity profiles that vary rapidly in space. Traditional internal mode solutions require discrete representations of velocity and therefore demand considerable resolution in high-gradient regions to accurately represent the velocity profile (we call these velocity solutions or VS). However, in contrast to velocity, shear stress profiles vary slowly over the depth in boundary layers. To take advantage of this, an alternate internal mode solution strategy has been developed that discretizes the shear stress (we call this a direct stress solution or DSS) [Luettich and Westerink, 1991; Luettich et al., 1994]. In shallow water a DSS can be accomplished quite efficiently using substantially fewer nodes than a VS (even when the latter is implemented on a stretched grid that is optimized for a logarithmic velocity profile). Comparisons between a 3-D DSS model and a 3-D VS model in the Bight of Abaco showed that when a no-slip bottom boundary condition was used, the VS (on a stretched grid) and the DSS (on a uniform grid) converged to essentially identical solutions, although the DSS required three to five nodes over the vertical, while the VS required 20-30 nodes over the vertical.

The derivation and behavior of the DSS internal mode equations are described in detail by Luettich et al. [1994]. Briefly, these equations are obtained by taking the vertical derivative of the traditional three-dimensional momentum equations (assuming a hydrostatic pressure distribution). If the standard eddy viscosity expression is inverted,

$$
\begin{aligned}
& \frac{\partial u}{\partial z}=\frac{\tau_{z x}}{E_{z} \rho_{0}} \\
& \frac{\partial v}{\partial z}=\frac{\tau_{z y}}{E_{z} \rho_{0}}
\end{aligned}
$$

where $u$ and $v$ are the depth-dependent velocity components and $E_{z}$ is the eddy viscosity; the vertical derivatives of velocity in the differentiated momentum equations can be replaced by shear stresses, yielding

$$
\begin{aligned}
& \frac{\partial}{\partial t}\left[\frac{\tau_{z x}}{E_{z} \rho_{0}}\right]-f \frac{\tau_{z y}}{E_{z} \rho_{0}}-\frac{(a-b)^{2}}{H^{2} \rho_{0}} \frac{\partial^{2} \tau_{z x}}{\partial \sigma^{2}}=A_{x} \\
& \frac{\partial}{\partial t}\left[\frac{\tau_{z y}}{E_{z} \rho_{0}}\right]+f \frac{\tau_{z x}}{E_{z} \rho_{0}}-\frac{(a-b)^{2}}{H^{2} \rho_{0}} \frac{\partial^{2} \tau_{z y}}{\partial \sigma^{2}}=A_{y}
\end{aligned}
$$

In (5a) and (5b), $A_{x}$ and $A_{y}$ represent the combined horizontal advective and diffusive terms, and a general terrain-following $\sigma$-coordinate system has been used in which $\sigma=a$ at the free surface and $\sigma=b$ at the bottom. Three different eddy viscosity formulations (with magnitudes dependent on the flow field) were used in this study.

For the present study the "local" form of the DSS equations is used in which the horizontal advective and diffusion terms are neglected in (5a) and (5b). Scaling arguments show that this is a reasonable assumption in shallow water when the rate of vertical momentum transport is much greater than the rate of horizontal momentum transport [Luettich et al., 1992].

Velocity is obtained from the solution for stress by integrating (4) from the bottom up

$$
\begin{aligned}
& u=u_{b}+\frac{H}{(a-b)} \int_{b}^{\sigma} \frac{\tau_{z x}}{E_{z} \rho_{0}} d \sigma \\
& v=v_{b}+\frac{H}{(a-b)} \int_{b}^{\sigma} \frac{\tau_{z y}}{E_{z} \rho_{0}} d \sigma
\end{aligned}
$$

where $u_{b}$ and $v_{b}$ are bottom slip velocities. A no-slip bottom boundary condition is implemented by setting $u_{b}$ and $v_{b}$ to zero. Alternatively, a slip condition can be implemented by relating $u_{b}$ and $v_{b}$ to the bottom stress using a standard quadratic expression

$$
\begin{aligned}
& u_{b}\left(u_{b}^{2}+v_{b}^{2}\right)^{1 / 2}=\frac{\tau_{b x}}{\rho_{0} C_{f 3 \mathrm{~d}}} \\
& v_{b}\left(u_{b}^{2}+v_{b}^{2}\right)^{1 / 2}=\frac{\tau_{b y}}{\rho_{0} C_{f 3 \mathrm{~d}}}
\end{aligned}
$$

where $C_{f 3 \mathrm{~d}}$ is the three-dimensional friction coefficient.

Equations (5a) and (5b) are solved using a Galerkin finite element method with linear basis functions in space and a finite difference method in time. The use of linear basis functions for $\tau_{z x}$ and $\tau_{z y}$, coupled with an eddy viscosity that is assumed to be piecewise linear over the water depth, allows a simple closed form solution to (6) and therefore facilitates the recovery of velocity from stress. The vertical differentiation that leads to (5a) and (5b) has the effect of removing the depth-averaged flow information from these equations. Therefore the internal mode solution is obtained 
by solving (5a) and (5b) simultaneously with the 2DDI momentum equations. We note that in the 3-D solution the momentum dispersion terms in the GWCE and 2DDI momentum equations are evaluated from the velocity profile information and are included in the computations. Also, in the 3-D solution the bottom stress terms in the GWCE and 2DDI momentum equations are evaluated explicitly and therefore (3a) and (3b) are not used.

\section{Model Runs}

The Bight of Abaco (Figure 1) is located on Little Bahama Bank in the northernmost portion of the Bahama Islands. The bight is approximately $100 \mathrm{~km}$ long by $40 \mathrm{~km}$ wide and is bounded by the island of Abaco to the south and east and by the islands of Little Abaco and Grand Bahama along the northern edge. The Northwest Providence Channel forms the western boundary.

The bathymetry of the bight is shown in Figure 1. Water depths are very shallow, ranging from 1 to $9 \mathrm{~m}$. A sill region of 2- to $5-\mathrm{m}$ depth exists immediately inside the ocean boundary, while a 7- to 9-m depression covers a significant portion of the northern region. The rapid drop off to water depths of $1000-2000 \mathrm{~m}$ immediately outside the bight ensures that shallow water tides within the bight are generated locally.

Bottom conditions also vary widely. The sill region is marked by sand waves, many of which are of the order of 1 to $3 \mathrm{~m}$ and by the presence of several small shoals. Small muddy mounds populate most of the northern reaches of the bight, while the southern region is characterized by a thin sediment cover over rock [Fillowx and Snyder, 1979]. From a modeling perspective this range of bed types could be represented by spatially variable friction coefficients, with higher values in the sill region and lower values in the interior sections of the domain. Reported data for different bottom types suggest that a value of $C_{f 2 \mathrm{~d}}$ in the range $0.006-0.01$ may be appropriate near the sill, while values in remaining areas are probably more typical, e.g., $C_{f 2 d}=0.003$ [Soulsby, 1990].

Filloux and Snyder [1979] collected detailed tidal elevation data at 25 locations within the bight (see Figure 1 for station locations). These have been analyzed for the $K_{1}$, $\mathrm{O}_{1}, \mathrm{~N}_{2}, \mathrm{M}_{2}$, and $\mathrm{S}_{2}$ astronomical constituents as well as for the $M_{4}$ and $M_{6}$ overtides. The elevation data were gathered over a sequence of three 1-month field studies, although only 15 pressure sensors were available at any one time. As a result, some of the stations were occupied on more than one occasion, and up to three amplitude and phase results were reported at these sites.

The variability of the amplitude measurements by Filloux and Snyder [1979] were reported as basin-wide, relative rms errors by W89. We have used the same amplitude error measure in this study

$$
S_{j}^{m}=\left\{\frac{\sum_{l=1}^{L^{\prime}} \sum_{k=1}^{K_{l}}\left[\zeta_{j}^{m}\left(x_{l}, k\right)-\frac{1}{K_{l}} \sum_{k=1}^{K_{l}} \zeta_{j}^{m}\left(x_{l}, k\right)\right]^{2}}{\sum_{l=1}^{L^{\prime}} \sum_{k=1}^{K_{1}}\left[\zeta_{j}^{m}\left(x_{l}, k\right)\right]^{2}}\right\}^{1 / 2}
$$

where $\zeta_{j}^{m}$ is the measured elevation amplitude component for the $j^{\text {th }}$ harmonic, $L^{\prime}$ is the number of stations with multiple measurements for a constituent, and $K_{l}$ is the number of measurement values at location $l$. In addition, we have developed a similar basin-wide, rms error for the phase measurements

$$
P_{j}^{m}=\left\{\frac{\sum_{l=1}^{L^{\prime}} \sum_{k=1}^{K_{l}}\left[\theta_{j}^{m}\left(x_{l}, k\right)-\frac{1}{K_{l}} \sum_{k=1}^{K_{l}} \theta_{j}^{m}\left(x_{l}, k\right)\right]^{2}}{K_{T}^{\prime}}\right\}^{1 / 2}
$$

where $\theta_{j}^{m}$ is the measured elevation phase component for the $j^{\text {th }}$ harmonic and $K_{T}^{\prime}$ is the total number of measurements from stations occupied more than once; i.e.,

$$
K_{T}^{\prime}=\sum_{l=1}^{L^{\prime}} K_{l} .
$$

These errors are listed in Table 1 and provide an experimental error baseline to be taken into account in the interpretation of model versus data comparisons. Unfortunately, no similar data set exists for tidal velocity.

The horizontal grid used in the model studies is shown in Figure 1. The grid consists of 926 nodes and 1696 elements and has a grid spacing that ranges from 0.8 to $2.8 \mathrm{~km}$. The average nodal spacing in the grid $(2.5 \mathrm{~km})$ is approximately half that used by S79 and W89. Preliminary grid convergence studies suggested that the present grid achieved a wellconverged solution.

Boundary conditions consisted of elevation amplitudes and phases interpolated from the observational data of Filloux and Snyder [1979]. Although previous studies in the bight have assumed that nonlinear waves are fully reflected at the ocean boundary (and thus have specified boundary conditions for astronomical constituents only), model results suggest that this assumption may not be entirely correct since both the $M_{4}$ and $M_{6}$ amplitudes were underpredicted near the ocean boundary. Sensitivity analyses conducted to examine the effect of open boundary conditions on the $M_{4}$ and $M_{6}$ constituents showed that agreement with data was marginally better when these constituents were specified. However, there was no change in the bias of the $M_{6}$ predictions. The ranges of the amplitudes and phases for the boundary forcings used in the model are listed in Table 2 .

Model results were analyzed using the least squares harmonic analysis method with up to 36 response frequencies (for the multiconstituent forcing). Basin-wide statistics of the model performance compared with the data were computed on a constituent by constituent basis.

For elevation amplitude a basin-wide, relative rms error between the model and the measured data was computed as

$$
S_{j}^{p}=\left\{\frac{\sum_{l=1}^{L} \sum_{k=1}^{K}\left[\zeta_{j}^{m}\left(x_{l}, k\right)-\zeta_{j}\left(x_{l}\right)\right]^{2}}{\sum_{l=1}^{L} \sum_{k=1}^{K}\left[\zeta_{j}^{m}\left(x_{l}, k\right)\right]^{2}}\right\}^{1 / 2}
$$

where $L$ is the total number of measurement sites, $K_{l}$ is the number of data points at site $l, \zeta_{j}^{m}$ is the measured elevation amplitude for the $j^{\text {th }}$ constituent, and $\zeta_{j}$ is the computed elevation amplitude for the $j^{\text {th }}$ constituent. 

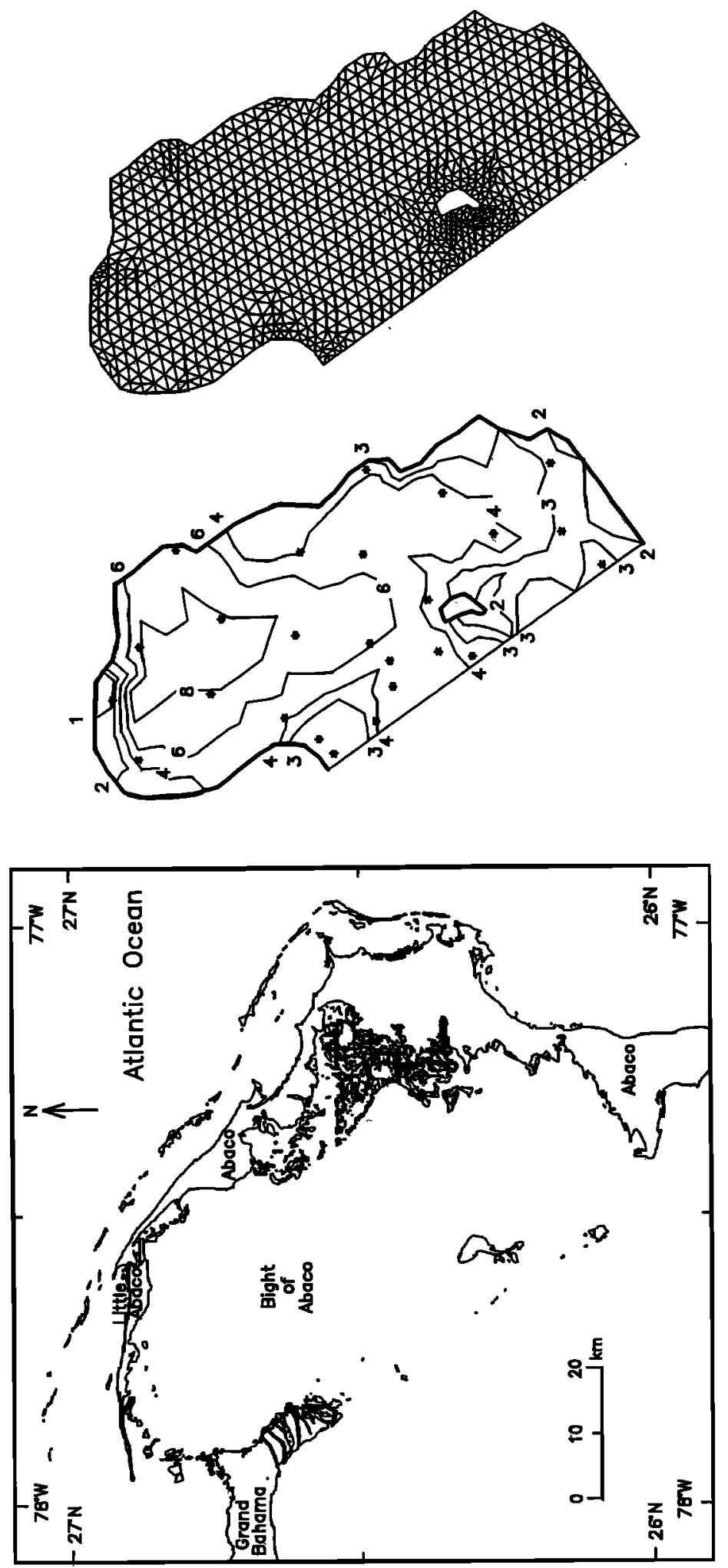

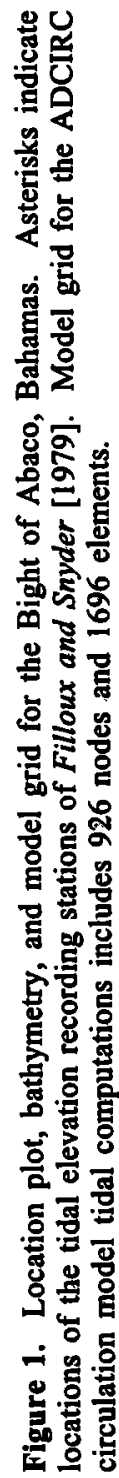


Table 1. Basin-wide Average Errors in Measured Elevation Amplitude and Phase

\begin{tabular}{lccccccc}
\hline & $\mathrm{O}_{1}$ & $\mathrm{~K}_{1}$ & $\mathrm{~N}_{2}$ & $\mathrm{M}_{2}$ & $\mathrm{~S}_{2}$ & $\mathrm{M}_{4}$ & $\mathrm{M}_{6}$ \\
\hline Amplitude, \% & 3.0 & 8.0 & 13.2 & 3.0 & 14.3 & 11.6 & 12.0 \\
Phase, deg & 2.3 & 5.2 & 11.2 & 3.3 & 10.4 & 9.8 & 12.9 \\
\hline
\end{tabular}

For elevation phase a basin-wide rms error between the model and the measured data was computed as

$$
P_{j}^{p}=\left\{\frac{\sum_{l=1}^{L} \sum_{k=1}^{K_{l}}\left[\theta_{j}^{m}\left(x_{l}, k\right)-\theta_{j}\left(x_{l}\right)\right]^{2}}{K_{T}}\right\}^{1 / 2}
$$

where $\theta_{j}^{m}$ is the measured elevation phase for the $j^{\text {th }}$ constituent, $\theta_{j}$ is the computed elevation phase for the $j^{\text {th }}$ constituent, and $K_{T}$ is the total number of measured values for all measurement sites. To complement these statistics, we also present the number of stations where the model overpredicts and underpredicts measured amplitudes and phases.

\section{2-D Model Runs}

In order to establish approximate friction parameters for subsequent runs with full constituent forcing, an initial set of runs was conducted with the 2-D model using the $M_{2}, M_{4}$, and $\mathrm{M}_{6}$ forcing and $C_{f 2 \mathrm{~d}}$ values ranging from 0.003 to 0.012 . Several values of $C_{f 2 \mathrm{~d}}$ were tested in this range, and a sample of the results is shown in Table 3. The results from these preliminary runs show that increasing the friction coefficient lowers the basin-wide elevation amplitude response of all constituents. Within the range of friction parameters considered, the lowest $\mathrm{M}_{2}$ elevation amplitude and phase prediction errors occurred when $C_{f 2 \mathrm{~d}}=0.0095$, which is consistent with the findings of S79 and W89. In addition, the $\mathrm{M}_{4}$ errors were also near their minimum for this value of $C_{f 2 d}$. However, neither the amplitude nor the phase errors were minimum for the $M_{6}$ constituent. Rather, the $M_{6}$ constituent was overpredicted by approximately $87 \%$.

It should be noted that the interaction of the $M_{2}$ constituent with itself through the quadratic friction term was the dominant nonlinearity responsible for the generation of the $M_{6}$ constituent. Trial runs with bottom friction as the

Table 2. Open Boundary Forcing Data

\begin{tabular}{ccc}
\hline Tide & $\begin{array}{c}\text { Amplitude, } \\
\mathrm{cm}\end{array}$ & $\begin{array}{c}\text { Phase, } \\
\text { deg }\end{array}$ \\
\hline $\mathrm{O}_{1}$ & $7.2-7.9$ & $202-212$ \\
$\mathrm{~K}_{1}$ & $8.6-10.0$ & $212-219$ \\
$\mathrm{~N}_{2}$ & $9.7-10.3$ & $341-10$ \\
$\mathrm{M}_{2}$ & $38.2-39.4$ & $6-14$ \\
$\mathrm{~S}_{2}$ & $5.6-6.3$ & $51-56$ \\
$\mathrm{M}_{4}$ & $0.2-0.3$ & $100-144$ \\
$\mathrm{M}_{6}$ & $0.2-0.3$ & $228-351$ \\
\hline
\end{tabular}

Phases are relative to the equilibrium tide at Greenwich. only nonlinear term produced an $M_{6}$ response essentiaily identical to that given above.

Given the approximate value of $C_{f 2 \mathrm{~d}}=0.0095$, a second set of runs was completed using all of the measured tidal constituents. Several values near $C_{f 2 \mathrm{~d}}=0.0095$ were considered, and in each case the model was run for 40 days, with a harmonic analysis of the last $\mathbf{3 0}$ days. The lowest $\mathbf{M}_{2}$ elevation errors were achieved when $C_{f 2 d}=0.0085$ (Table 4). Although the amplitude error was identical at $C_{f 2 d}=0.009$, the distribution of the error was somewhat worse. As noted in the introduction, the reduction in the optimum friction factor from the initial set of runs reflects the contribution of the secondary constituents to the frictional balance in the bight, as the secondary waves provide some complementary damping of the $M_{2}$ constituent through the quadratic friction term.

In general, the elevation amplitude errors for the $O_{1}, K_{1}$, $S_{2}$, and $M_{4}$ tides are reduced as the friction factor is

Table 3. 2-D Model Results, $M_{2}, M_{4}$, and $M_{6}$ Forcing

\begin{tabular}{|c|c|c|c|}
\hline Measure & $\mathbf{M}_{2}$ & $\mathbf{M}_{4}$ & $\mathbf{M}_{6}$ \\
\hline \multicolumn{4}{|c|}{$C_{f 2 d}=0.003$} \\
\hline Average amplitude, $m$ * & 0.2906 & 0.0138 & 0.0121 \\
\hline Amplitude error, $\%$ & 49.3 & 99.9 & 140.7 \\
\hline Phase error, deg & 11.0 & 35.6 & 28.2 \\
\hline Amplitude overpredicted & 20 & 20 & 20 \\
\hline Amplitude underpredicted & 4 & 4 & 4 \\
\hline Phase overpredicted & 10 & 6 & 6 \\
\hline Phase underpredicted & 14 & 19 & 19 \\
\hline \multicolumn{4}{|c|}{$C_{f 2 d}=0.006$} \\
\hline Average amplitude, $m^{*}$ & 0.2310 & 0.0093 & 0.0108 \\
\hline Amplitude error, \% & 18.1 & 36.2 & 110.0 \\
\hline Phase error, deg & 6.7 & 26.0 & 27.6 \\
\hline Amplitude overpredicted & 19 & 18 & 21 \\
\hline Amplitude underpredicted & 5 & 6 & 3 \\
\hline Phase overpredicted & 11 & 11 & 7 \\
\hline Phase underpredicted & 13 & 14 & 18 \\
\hline \multicolumn{4}{|c|}{$C_{f 2 d}=0.0095$} \\
\hline Average amplitude, $\mathrm{m}^{*}$ & 0.1998 & 0.0074 & 0.0100 \\
\hline Amplitude error, \% & 7.7 & 22.4 & 87.4 \\
\hline Phase error, deg & 5.3 & 25.7 & 30.0 \\
\hline Amplitude overpredicted & 11 & 12 & 21 \\
\hline Amplitude underpredicted & 13 & 11 & 3 \\
\hline Phase overpredicted & 15 & 12 & 9 \\
\hline Phase underpredicted & 9 & 13 & 16 \\
\hline \multicolumn{4}{|c|}{$c_{f 2 d}=0.012$} \\
\hline Average amplitude, $\mathrm{m}^{*}$ & 0.1860 & 0.0067 & 0.0096 \\
\hline Amplitude error, \% & 10.8 & 26.8 & 76.4 \\
\hline Phase error, deg & 5.4 & 26.5 & 32.1 \\
\hline Amplitude overpredicted & 4 & 8 & 21 \\
\hline Amplitude underpredicted & 20 & 16 & 3 \\
\hline Phase overpredicted & 17 & 12 & 10 \\
\hline Phase underpredicted & 7 & 13 & 15 \\
\hline
\end{tabular}

$C_{f 2 \mathrm{~d}}$ is the two-dimensional friction coefficient.

* Steady (zero-frequency) values of average ainplitude are as follows: for $C_{f 2 \mathrm{~d}}=0.003,0.0214$; for $C_{f 2 \mathrm{~d}}=0.006,0.0207$; for $C_{f 2 \mathrm{~d}}=0.0095,0.0205$; and for $C_{f 2 \mathrm{~d}}=0.012,0.0204$. 
decreased, while the errors for the $\mathrm{N}_{2}$ and $\mathrm{M}_{6}$ tides increase with decreasing friction factor. A marked improvement occurred in the $M_{6}$ constituent versus the initial set of runs (the amplitude error is reduced from 87.4 to $35.5 \%$ ) due to the addition of the secondary astronomical constituents. Comparing Table 1 and Table 4 shows that the basin-wide amplitude and phase errors for all but the $K_{1}$ constituent were significantly larger than the average observational variability. Also, Table 4 shows that the distribution of errors was toward overdamping for the $O_{1}, K_{1}$, and $S_{2}$ constituents and underdamping for the $\mathrm{M}_{6}$ and $\mathrm{N}_{2}$ constituents.

The $\mathrm{M}_{6}$ amplitude error was significantly lower than the final optimum result of W89 (35.5 versus 53\%). The most likely cause for this improvement is that the time-stepping model contains a more complete representation of the nonlinear constituent interactions than the harmonic in time model used by W89. Small improvements may also be due to the inclusion of $M_{6}$ boundary forcing and to the refined grid used in the present study.

Computed depth-averaged $M_{2}$ tidal current ellipses for the 2-D run using $C_{f 2 \mathrm{~d}}=0.0085$ are shown in Figure 2 . Currents are strongest in the sill region, where speeds may exceed 50 $\mathrm{cm} / \mathrm{s}$ and are considerably weaker in the deeper basin. In addition, the ellipses are highly rectilinear, a feature important to the frictional processes occurring in the bight (see later discussion).

The value of $C_{f 2 \mathrm{~d}}=0.0085$ obtained with the full set of tidal constituents is high in comparison with values typically used in tidal modeling studies. Two possible explanations for this high value were identified. First, as noted above, the value of $C_{f 2 \mathrm{~d}}=0.0085$ may actually be a reasonable choice for the sill region which is characterized by sand waves, although it is probably not representative of the bottom features found in the northern depression or the eastern and southern sections of the bight. Second, the presence of sand waves introduces a large amount of uncertainty in the bathymetric depth in the sill region. Since the continuity equation only requires the correct horizontal flux $(U H, V H)$, it may be possible to obtain a reasonable calibration to $M_{2}$ surface elevation data despite having incorrect bathymetric depths, provided the depth-averaged velocities are also incorrect (e.g., if $h$ is too large, $U$ and $V$ must be too small). In the momentum equation the bottom friction term (equation (3)) behaves as $U^{2} / H$ and therefore will also be affected by incorrect bathymetry (e.g., if $h$ is too large, the bottom friction term will be too small). This can be compensated for, however, using the friction coefficient $C_{f 2 d}$ (e.g., if $h$ is too large, an erroneously high value of $C_{f 2 d}$ will be required to calibrate the model).

To test these ideas, we first made a series of runs using the multiconstituent forcing and a spatially varying friction factor. Typically, three friction factor regions were selected to allow a high friction factor in the sill region, more traditional values (e.g., $C_{f 2 \mathrm{~d}}=0.003$ ) in the northern and eastern parts of the bight, and a narrow band of medium values as a transition. In general, we found that most of the dissipation of the astronomical constituents and most of the nonlinear interactions occur in the areas where velocities are highest, i.e., the sill region. The effect of frictional processes (e.g., $M_{6}$ production) and hence sensitivity to bottom friction parameters is greatly reduced in other areas of the bight where currents are weak. Consequently, a spatially variable friction factor or, in the 3-D case, a spatially varying bottom roughness can be used but has a minimal effect on the overall model results.

In a second series of runs we used a friction factor of $C_{f 2 d}$ $=0.003$ and reduced the water depths. The results indicated that a successful $M_{2}$ calibration could be achieved by reducing the depth by approximately one third. However, this caused the basin-wide error in the $\mathrm{M}_{4}$ overtide to triple $\left(\mathrm{M}_{4}\right.$ was substantially overpredicted throughout the domain) and therefore cannot be considered a viable explanation for the high friction coefficient. Additional sensitivity runs showed that the overtides were much more sensitive than astronomical tides to changes in the depth distribution. Overall, the original depth distribution produced the smallest

Table 4. 2-D Runs With All Constituents and Full Nonlinear Tidal Interactions

\begin{tabular}{|c|c|c|c|c|c|c|c|}
\hline Measure & $\mathbf{O}_{1}$ & $\mathbf{K}_{1}$ & $\mathbf{N}_{2}$ & $\mathbf{M}_{2}$ & $\mathbf{S}_{2}$ & $\mathbf{M}_{4}$ & $M_{6}$ \\
\hline \multicolumn{8}{|c|}{$C_{f 2 d}=0.009$} \\
\hline Amplitude error, \% & 7.3 & 9.4 & 19.6 & 7.7 & 19.4 & 23.1 & 33.6 \\
\hline Phase error, deg & 4.9 & 7.4 & 13.1 & 5.3 & 15.9 & 25.8 & 27.9 \\
\hline Amplitude overpredicted & 3 & 5 & 15 & 9 & 3 & 10 & 20 \\
\hline Amplitude underpredicted & 21 & 19 & 9 & 15 & 21 & 13 & 4 \\
\hline Phase overpredicted & 20 & 20 & 19 & 16 & 19 & 14 & 7 \\
\hline Phase underpredicted & 5 & 5 & 6 & 9 & 6 & 11 & 18 \\
\hline \multicolumn{8}{|c|}{$C_{f 2 d}=0.0085$} \\
\hline Amplitude error, $\%$ & 6.2 & 8.6 & 20.2 & 7.7 & 18.9 & 21.9 & 35.5 \\
\hline Phase error, deg & 4.5 & 7.0 & 13.0 & 5.4 & 15.8 & 25.7 & 27.7 \\
\hline Amplitude overpredicted & 6 & 6 & 15 & 13 & 4 & 10 & 20 \\
\hline Amplitude underpredicted & 18 & 18 & 9 & 11 & 20 & 12 & 4 \\
\hline Phase overpredicted & 20 & 20 & 19 & 15 & 19 & 14 & 7 \\
\hline Phase underpredicted & 5 & 5 & 6 & 10 & 6 & 11 & 18 \\
\hline \multicolumn{8}{|c|}{$C_{f 2 d}=0.008$} \\
\hline Amplitude error, $\%$ & 5.3 & 8.1 & 21.0 & 8.2 & 18.5 & 21.3 & 37.3 \\
\hline Phase error, deg & 4.2 & 6.7 & 12.9 & 5.4 & 15.8 & 25.5 & 27.4 \\
\hline Amplitude overpredicted & 7 & 7 & 15 & 16 & 7 & 12 & 20 \\
\hline Amplitude underpredicted & 17 & 17 & 9 & 8 & 16 & 12 & 4 \\
\hline Phase overpredicted & 19 & 19 & 18 & 14 & 18 & 14 & 7 \\
\hline Phase underpredicted & 6 & 6 & 7 & 11 & 7 & 11 & 18 \\
\hline
\end{tabular}




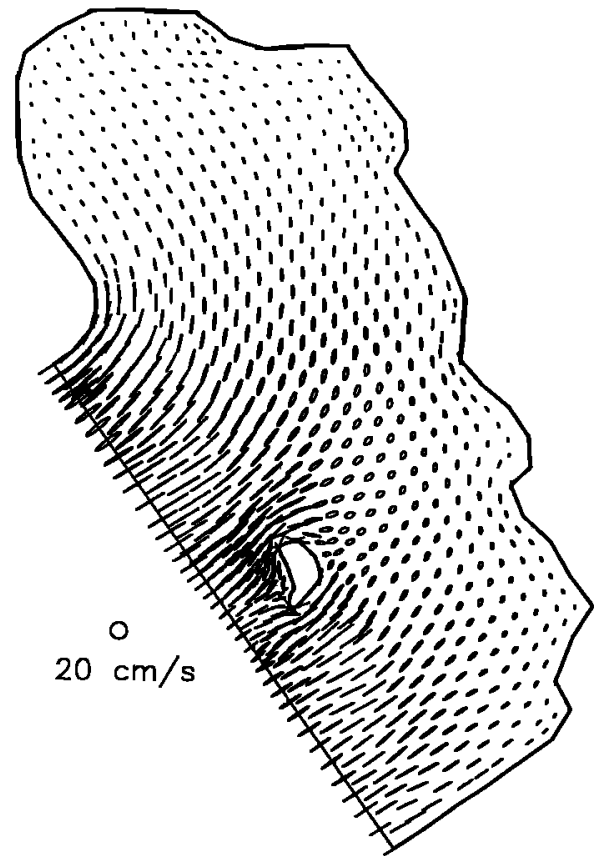

Figure 2. Depth-averaged $M_{2}$ current ellipse for 2-D run using $C_{f 2 \mathrm{~d}}=0.0085$.

errors in the overtides. On the basis of these findings we retain a constant friction coefficient or roughness height throughout the domain that is most representative of the sill region, and we use depths as originally reported by Filloux and Snyder [1979].

\section{3-D Model Runs}

Given this baseline of 2-D model results, we next consider the response of the 3-D model. While frictional effects in the 2-D model are represented solely by the bottom stress parameterization, in the 3-D model they are contained in the eddy viscosity term and in the bottom boundary condition. A variety of eddy viscosity formulations have been proposed in the literature. We considered three of these in our 3-D model runs as follows: case 1 is $E_{z}=A_{1} H\left(U^{2}+V^{2}\right)^{1 / 2}$, constant over the water column; case 2 is $E_{z}=A_{2}\left(U^{2}+V^{2}\right)$, constant over the water column; and case 3 is $E_{z}=\kappa u_{*} z_{0}$ at the bottom, $E_{z}=\kappa_{*}\left(h+z+z_{0}\right)$ over the lower $20 \%$ of the water column, and $E_{z}=0.2 \kappa_{*} H$ over the upper $80 \%$ of the water column.

Here $A_{1}$ and $A_{2}$ are free coefficients, $u_{*}$ is the shear velocity, $z_{0}$ is the bottom roughness, and $x=0.41$. The parameter $A_{2}$ is typically expressed as $A_{2}=K_{1} / \omega_{1}$, where $K_{1}$ is a dimensionless coefficient (sometimes taken as $2.0 \times 10^{-5}$ ) and $\omega_{1}$ is a characteristic frequency of the order of $1.0 \times 10^{-4}$ [Davies and Aldridge, 1993].

Although eddy viscosities that are constant over the depth are frequently used in modeling studies, they are incapable of representing the bottom boundary layer in a physically realistic manner. Therefore, when this type of eddy viscosity is used, it is appropriate to parameterize the boundary layer using a bottom slip condition. The case 1 formulation is useful in areas where the bottom boundary layer thickness is limited by the water depth [Davies and Aldridge, 1993]. while the case 2 formulation is typically used in deep water where boundary layers are rotationally limited [Davies and Furnes, 1980]. Although case 1 is more appropriate in the shallow waters of the Bight of Abaco, both formulations were considered to determine the effects on model response. In either case, two parameters ( $A_{1}$ or $A_{2}$ and the slip coefficient $\left.C_{f 3 \mathrm{~d}}\right)$ are introduced into the model.

A unification of case 1 and case 2 formulations has been presented by Aldridge and Davies [1993], in which $H$ in case $I$ is replaced by $\Delta$, a length scale proportional to $u_{*}$, when $\Delta$ is less than $H$. This single formulation may be appropriate in both shallow and deep water since the smaller-scale $\Delta$ is used when the boundary layer does not extend over the entire depth. While this formulation could be used in the present study, its effect would be essentially equivalent to case 1 due to the shallow depths found in the bight.

The case 3 formulation is based on observational and theoretical studies of boundary layers which suggest the eddy viscosity is proportional to $\kappa u_{*} z$ near the bottom. The eddy viscosity relationship used in case 3 is based on measurements made in tidal currents [Bowden et al., 1959] and is consistent with the use of a no-slip condition at the bottom. One advantage of the DSS technique is that $u_{*}$ is derived directly from the computed bottom stress, rather than the velocity profile. (The importance of high vertical resolution for computing shear-velocity-dependent eddy viscosity in a VS model was demonstrated by Davies [1993b]). Using this eddy viscosity formulation, only one parameter (the bottom roughness) is introduced into the model.

In the comparisons which follow we selected 3-D model parameters to produce $M_{2}$ elevation amplitudes as close as possible to our "best" 2-D response (using $C_{f 2 \mathrm{~d}}=0.0085$ ) and then concentrated on the associated responses at other frequencies. In case 1 and case 2 the parameters $A_{1}$ and $A_{2}$ may be applied in different combinations with $C_{f 3 d}$ to yield very similar elevation responses. These combinations primarily affect the water column shear, so without measured velocity profiles it is impossible to reduce the possibilities further. However, knowing that these eddy viscosity formulations are inappropriate for boundary layer flows, we attempted to minimize the presence of a bottom boundary layer by using the lowest slip coefficient possible. Model tests indicated that $C_{f_{3 \mathrm{~d}}}$ values less than 0.01 were unable to provide proper damping of the $M_{2}$ constituent as it propagated over the sill region.

With $C_{f 3 d}$ established we then calibrated the models by varying $A_{1}$ or $A_{2}$. The closest agreement between 2-D and 3$D$ models was achieved using a slip coefficient of $C_{f 3 \mathrm{~d}}=0.01$ together with $A_{1}=0.038$ (case 1) and $A_{2}=0.4 \mathrm{~s}$ (case 2). Since these calibrated values of $A_{1}$ and $A_{2}$ are higher than those typically used (and $C_{f 3 \mathrm{~d}}$ is nearly equal to $C_{f 2 \mathrm{~d}}$ ), one may expect vertical shear to be near zero. This fact suggests that the 3-D models will behave essentially as the 2-D model. However, some differences in the overtides were observed, and, as shall be discussed, these differences are related to the friction formulations used in the models.

For 3-D case 3 the bottom roughness $z_{0}$ was adjusted to produce similar 2-D and 3-D $\mathrm{M}_{2}$ elevation results; the calibrated roughness value was $z_{\mathrm{o}}=1.4 \mathrm{~cm}$. Using the relation for rough turbulent flow $\left(z_{0}=k_{s} / 30\right), z_{0}$ corresponds to an equivalent Nikuradse diameter $k_{s}$ of $0.4 \mathrm{~m}$, approximately the size of the sand waves in the sill region. Spatially variable roughness values were not examined, as 
results from the 2-D runs indicate model response is insensitive to friction parameters in areas away from the sill.

\section{2-D/3-D Model Comparisons}

The results from the 2-D and 3-D runs are compared in Figures 3-5. In each of the figures, elevation amplitudes from the 3-D model are plotted against elevation amplitudes from the 2-D model for all nodes in the domain. There is essentially no difference in the 2-D and 3-D, case 1 model results (Figure 3), and the plots for both astronomical and overtide constituents are virtually straight lines. The secondary astronomical constituents in the 3-D, case 2 model are similar to those in the 2-D model (Figure 4). However, the $M_{2}$ overtides in the 3-D, case 2 model are systematically larger than their 2-D counterparts. The secondary astronomical constituents in the $3-\mathrm{D}$, case 3 model also have the same basic behavior as the 2-D model (Figure 5). The largest differences between the 3-D, case 3 model and the 2-D model again occur in the overtide constituents. The $M_{4}$ response is consistently higher in the 3-D model, while the $M_{6}$ response, though similar, exhibits significantly more scatter and a slight reduction, on average.

The comparisons presented above are supported by the 3-D results included in Table 5 . As shown in Table 5 , the amplitude errors for the 3-D, case 1 model are nearly identical to those for the optimal 2-D run (Table 4). In the 3-D, case 2 result the $M_{6}$ amplitude error is significantly larger than in the 2-D model ( 52.8 versus $35.5 \%$ ) and the $\mathrm{M}_{4}$ amplitude is overpredicted at five more stations. The 3-D, case 3 model produced a small increase in the $\mathbf{M}_{4}$ elevation amplitude error as well as a greater number of overpredicted stations. The overall $\mathbf{M}_{6}$ elevation amplitude error in this case is quite
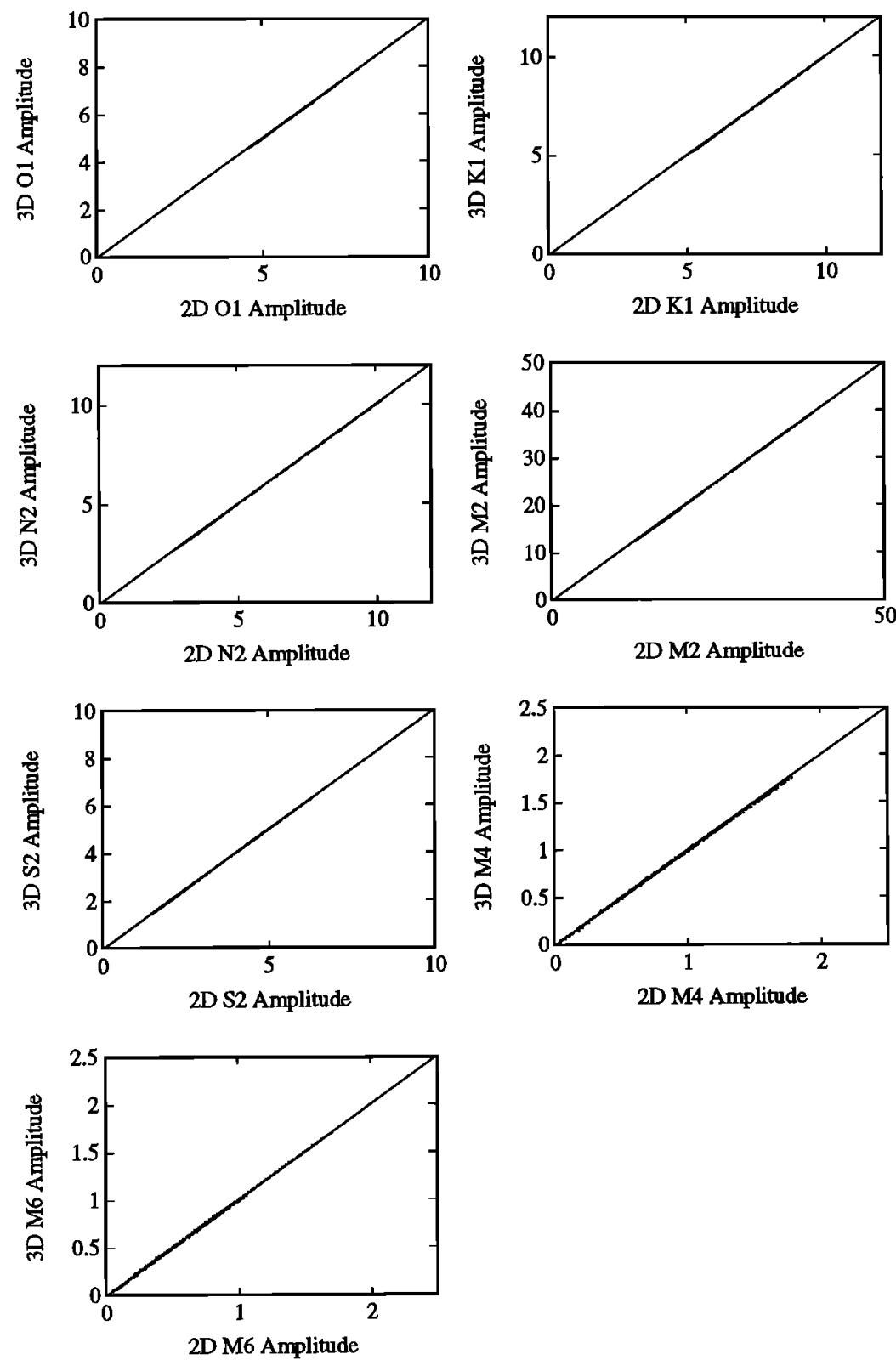

Figure 3. Results for 3-D, eddy viscosity case 1 run versus 2-D run. Results are plotted at all nodes in the domain. 

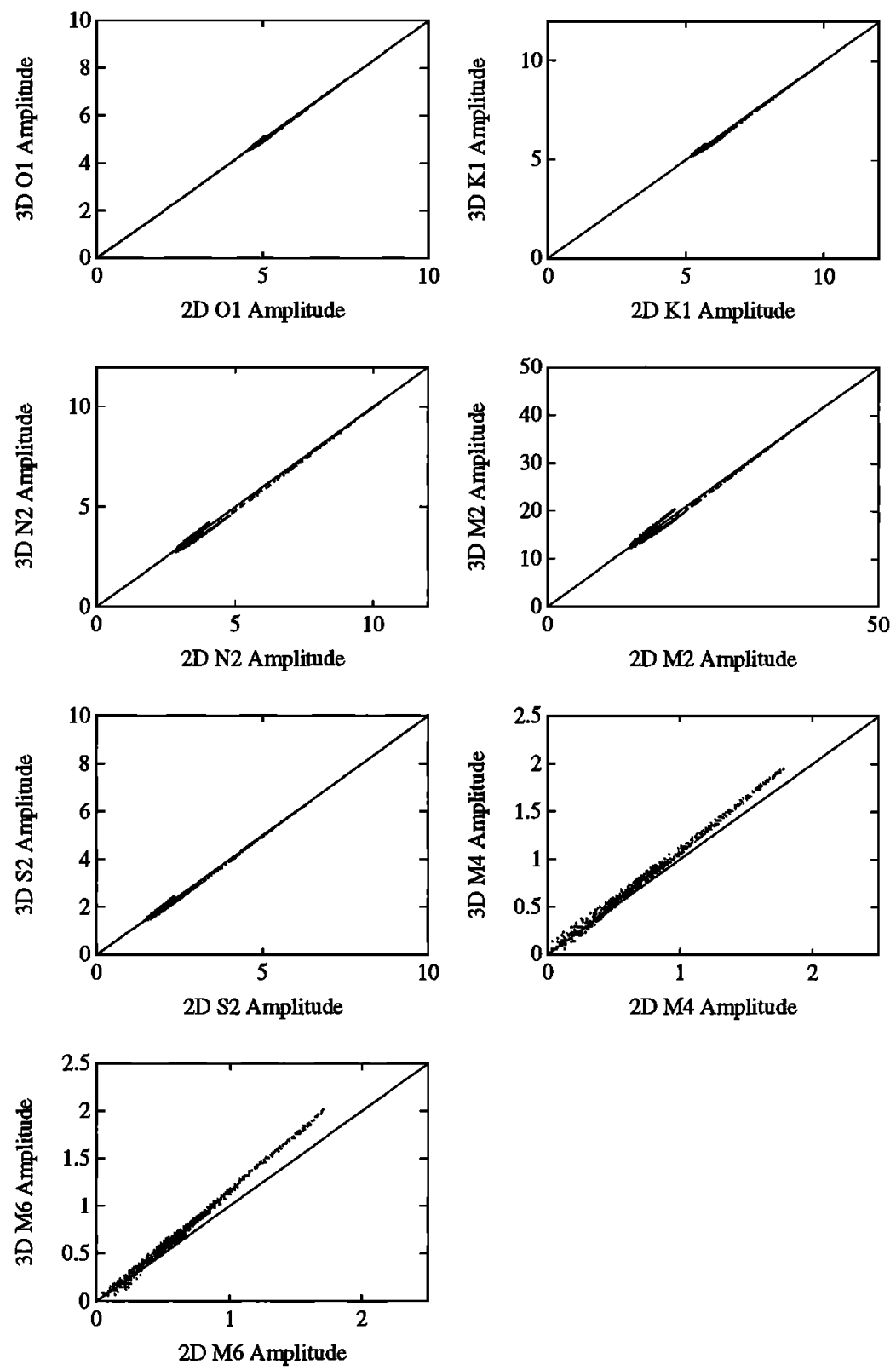

Figure 4. Results for 3-D, eddy viscosity case 2 run versus 2-D run. Results are plotted at all nodes in the domain.

close to the 2-D error. In general, phases are less sensitive than amplitudes to the various model formulations.

In summary, although the 2-D and 3-D models can be calibrated to produce nearly identical $M_{2}$ responses, there are systematic differences in the overtides and slight differences in the secondary astronomical tides. Furthermore, the results indicate that the larger overtide amplitudes predicted by the 3-D model are further away from the measured data than the 2$D$ model results. Therefore the over- and underprediction errors encountered in the Bight of Abaco for the 2-D model persist in the 3-D model, and in some cases these errors may be worse.

\section{Friction Analysis}

Since friction is the dominant nonlinearity in the Bight of Abaco, we seek further insight into the nonlinear tidal dynamics by analyzing the friction terms in the 2-D and 3-D models in detail. This is done using the harmonic approach of S79 and Walters [1986, 1987].

\section{2-D Model Equations}

Velocity and elevation in the 2-D model equations can be expressed as a harmonic series

$$
\begin{gathered}
U(x, t)=U_{0}(x)+\frac{1}{2} \sum_{\substack{n=-N \\
n \neq 0}}^{N} U_{n}(x) \exp \left(-i \omega_{n} t\right) \\
\zeta(x, t)=\zeta_{0}(x)+\frac{1}{2} \sum_{\substack{n=-N \\
n \neq 0}}^{N} \zeta_{n}(x) \exp \left(-i \omega_{n} t\right)
\end{gathered}
$$

where $N$ is the number of tidal constituents, $\omega_{n}$ is the 

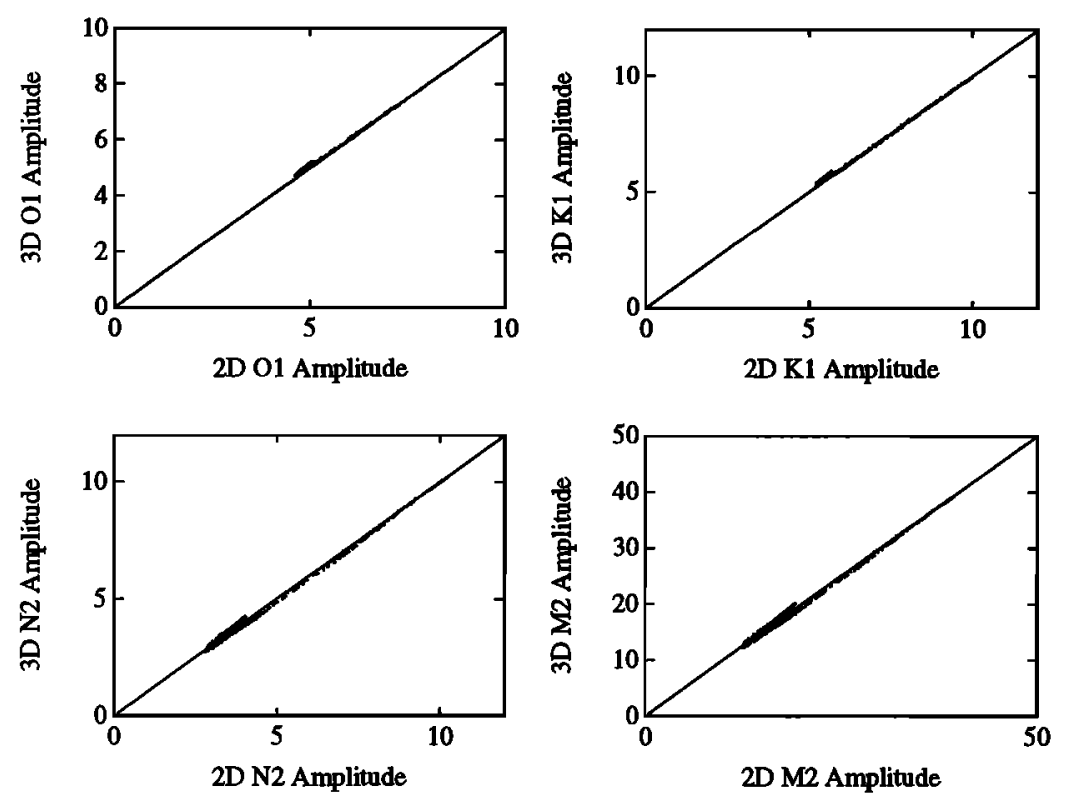

2D M2 Amplitude
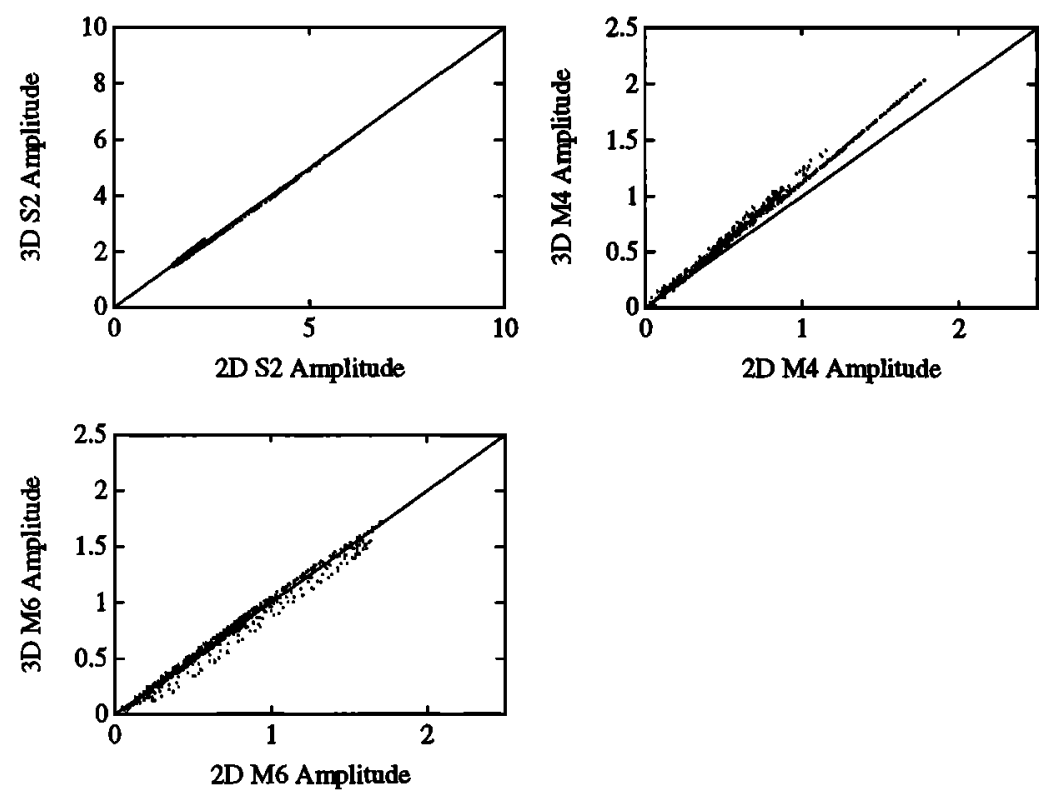

Figure 5. Results for 3-D, eddy viscosity case 3 run versus 2-D run. Results are plotted at all nodes in the domain.

angular frequency for the $n^{\text {th }}$ constituent, and $\omega_{-n}=-\omega_{n}$. $U_{0}(x)$ and $\zeta_{0}(x)$ are the steady components and $U_{n}(x)$ and $\zeta_{n}(x)$ are complex harmonic amplitudes for the depthaveraged velocity vector and surface elevation, respectively.

The $U|U|$ term in the quadratic friction relation, (3), is evaluated by separating the absolute value of $U$ into timedependent and time-independent parts and expanding in a Taylor series about the time-independent part. Similarly, the $1 / H=1 /(h+\zeta)$ that appears in the bottom stress term in the 2-D equations can be expanded in a Taylor series about the bathymetric depth. After applying a harmonic decomposition, the resulting expression for the $n^{\text {th }}$ constituent of the bottom stress term is

$$
\left(\frac{\tau_{b}}{H \rho_{\mathrm{o}}}\right)_{n}=\tau_{b 2 \mathrm{D}}+\left(\tau_{b 2 \mathrm{D}}^{v}+\tau_{b 2 \mathrm{D}}^{\nu^{\prime}}+\ldots\right)+\left(\tau_{b 2 \mathrm{D}}^{\zeta}+\ldots\right)
$$

where $\tau_{b 2 \mathrm{D}}$ is a linear stress term and the superscripts $\nu$ and $\zeta$ denote terms nonlinear in velocity and elevation as discussed below (see S79 and Walters and Werner [1991] for details).

The leading order contributor to the bottom stress term in (14) is linear in velocity and independent of surface elevation

$$
\tau_{b 2 \mathrm{D}} \equiv \frac{C_{f 2 \mathrm{~d}} \lambda}{h} U_{n}
$$

where

$$
\lambda=\left(\frac{1}{4} \sum_{\substack{n=-N \\ n \neq 0}}^{N} U_{n} \cdot U_{n}^{*}+U_{0} \cdot U_{0}\right)^{1 / 2}
$$


Table 5. 3-D Model Prediction Errors

\begin{tabular}{|c|c|c|c|c|c|c|c|}
\hline Measure & $\mathrm{O}_{1}$ & $\mathbf{K}_{\mathbf{1}}$ & $\mathbf{N}_{2}$ & $\mathbf{M}_{2}$ & $\mathbf{S}_{2}$ & $\mathbf{M}_{4}$ & $\mathbf{M}_{6}$ \\
\hline \multicolumn{8}{|c|}{ Case I } \\
\hline Amplitude error, $\%$ & 6.4 & 8.7 & 20.0 & 7.7 & 19.0 & 22.0 & 35.6 \\
\hline Phase error, deg & 4.4 & 6.9 & 12.9 & 5.4 & 15.8 & 25.5 & 27.9 \\
\hline Amplitude overpredicted & 6 & 5 & 15 & 13 & 4 & 10 & 20 \\
\hline Amplitude underpredicted & 18 & 19 & 9 & 11 & 20 & 12 & 4 \\
\hline Phase overpredicted & 20 & 19 & 18 & 14 & 17 & 14 & 7 \\
\hline Phase underpredicted & 5 & 6 & 7 & 11 & 8 & 11 & 18 \\
\hline \multicolumn{8}{|c|}{ Case 2} \\
\hline Amplitude error, \% & 6.0 & 8.7 & 21.3 & 8.9 & 20.1 & 22.8 & 52.8 \\
\hline Phase error, deg & 4.5 & 6.6 & 12.8 & 5.8 & 16.0 & 25.7 & 26.2 \\
\hline Amplitude overpredicted & 5 & 5 & 13 & 14 & 5 & 15 & 21 \\
\hline Amplitude underpredicted & 19 & 19 & 11 & 10 & 19 & 9 & 3 \\
\hline Phase overpredicted & 20 & 21 & 16 & 13 & 18 & 14 & 9 \\
\hline Phase underpredicted & 5 & 4 & 9 & 12 & 7 & 11 & 16 \\
\hline \multicolumn{8}{|c|}{ Case 3} \\
\hline Amplitude error, \% & 5.1 & 8.2 & 21.5 & 8.5 & 19.7 & 25.5 & 35.7 \\
\hline Phase error, deg & 4.2 & 6.4 & 12.8 & 5.7 & 16.0 & 26.2 & 25.9 \\
\hline Amplitude overpredicted & 8 & 7 & 15 & 15 & 5 & 15 & 20 \\
\hline Amplitude underpredicted & 16 & 17 & 9 & 9 & 19 & 8 & 4 \\
\hline Phase overpredicted & 18 & 21 & 18 & 14 & 17 & 14 & 10 \\
\hline Phase underpredicted & 7 & 4 & 7 & 11 & 8 & 11 & 15 \\
\hline
\end{tabular}

is the rms velocity and $U_{n}^{*}$ is the complex conjugate of $U_{n}$. In the Bight of Abaco, typical rms velocities in the sill region are approximately $25-30 \mathrm{~cm} / \mathrm{s}$.

The first two nonlinear terms resulting from the quadratic friction relation are

$$
\begin{gathered}
\tau_{b 2 \mathrm{D}}^{\nu} \equiv \frac{C_{f 2 \mathrm{~d}}}{8 h \lambda} \sum_{\substack{p, q, r \\
p \neq-q}}\left(U_{p} \cdot U_{q}\right) U_{r} \\
\tau_{b 2 \mathrm{D}}^{v^{\prime}} \equiv-\frac{C_{f 2 \mathrm{~d}}}{128 h \lambda^{3}} \sum_{\substack{p, q, r, s, t \\
p \neq-q \\
r \neq-s}}\left(U_{p} \cdot U_{q}\right)\left(U_{r} \cdot U_{s}\right) U_{t}
\end{gathered}
$$

while the leading nonlinear finite amplitude term is

$$
\tau_{b 2 \mathrm{D}}^{\zeta} \equiv-\frac{C_{f 2 \mathrm{~d}} \lambda}{h^{2}} \sum_{p, q} \zeta_{p} U_{q}
$$

The indices $p, q, r, s, t$ in (17)-(19) are any integers that satisfy $\omega_{p}+\omega_{q}+\omega_{r}=\omega_{n}, \omega_{p}+\omega_{q}+\omega_{r}+\omega_{s}+\omega_{t}=\omega_{n}$, and $\omega_{p}+\omega_{q}=\omega_{n}$, respectively.

The first nonlinear quadratic friction term (17) is responsible for many of the overtides and compound tidal interactions in the Bight of Abaco. It is the primary generator of the $\mathbf{M}_{6}$ overtide through the interaction of the dominant $\mathrm{M}_{2}$ tide with itself, and it is also an important contributor to the damping of secondary astronomical constituents through the interactions of the $\mathbf{M}_{2}$ tide with those constituents. The second nonlinear quadratic friction term, (18), adds destructively to contributions of the first nonlinear quadratic friction term at the same frequency; the reduction in the $M_{6}$ overtide witnessed by W89 due to $M_{2}-N_{2}$ and $M_{2}-S_{2}$ interactions is an example of this effect. The finite amplitude nonlinearity, (19), is responsible for a portion of the $M_{4}$ overtide through the interaction of the $M_{2}$ amplitude and the $\mathbf{M}_{2}$ current. The finite amplitude term in the continuity equation and the advective term in the momentum equation (to a much lesser extent in the Bight of Abaco) also contribute. The ratio of the first nonlinear quadratic friction term (17) to the linear friction term (15) (and therefore the rate of $M_{6}$ generation) scales with $1 / 8 \lambda^{2}$. The ratio of the finite amplitude friction term (19) to the linear friction term (and therefore the rate of $\mathrm{M}_{4}$ generation) scales with $1 / h$.

The influence of the lowest-order nonlinear quadratic friction term (17) on each of the astronomical constituents can be estimated by considering its magnitude relative to the linear term (15). A plot of the ratio for the $\mathrm{M}_{2}$ constituent at every node in the model domain for the optimal 2-D run is shown in Figure 6. The ratio is plotted versus the ellipticity of the $\mathrm{M}_{2}$ tidal current, $\phi,(\phi=1-B / A$, where $A$ and $B$ are the lengths of the major and minor ellipse axes, respectively) computed from model results (see Figure 2). The dashed line represents the value of this ratio if only an $\mathrm{M}_{2}$ tide were present. For the $\mathrm{M}_{2}$-only case the ratio has a minimum value of zero for circular current ellipses and a maximum value of $1 / 4$ for rectilinear ellipses. The ratios in the bight are consistently above this $M_{2}$-only case, indicating that interactions involving secondary astronomical constituents increase the nonlinear term relative to their effect on the linear term through the rms velocity. Overall, the bight is characterized by near-rectilinear current ellipses $(\phi \geq 0.7)$ and ratio values between 0.3 and 0.35 . A similar plot has been made for a typical secondary astronomical constituent (the $O_{1}$ ) in Figure 6. This ratio generally varies between 0.7 and 1 and is typical of the values of the other secondary astronomical constituents.

The significance of these results is that the nonlinear contribution of (17) to the total friction acting on the $\mathrm{M}_{2}$ constituent is only $30-35 \%$ of the linear contribution. However, for secondary constituents the nonlinear friction term (17) and the linear friction term are almost of equal importance. Thus the damping of the $\mathrm{M}_{2}$ constituent is due primarily to the linear component of friction, while the damping of secondary constituents is influenced by the nonlinear and the linear terms in nearly equal proportion. 

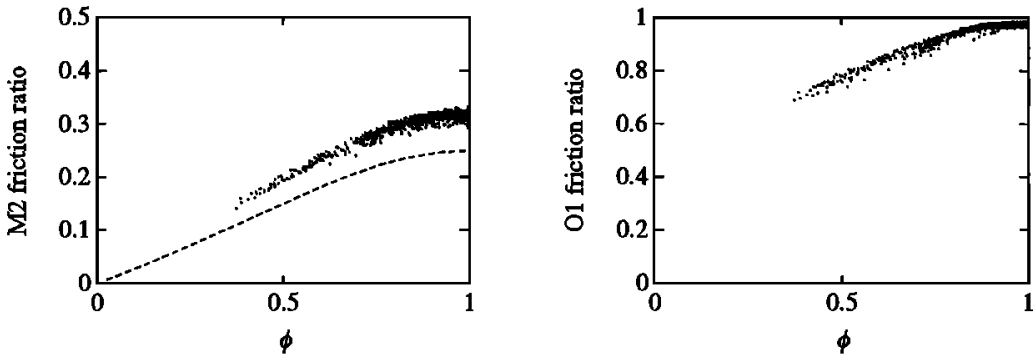

Figure 6. (left) Ratio of the first nonlinear quadratic friction term to the linear friction term for the $\mathrm{M}_{2}$ constituent versus $\phi$ for the $M_{2}$ constituent. Note that $\phi=1$ for rectilinear currents and $\phi=0$ for circular currents. Dots indicate ratios computed using all astronomical tides (from the optimal 2-D run), dashed line indicates the limit for ratio computed using only the $\mathrm{M}_{2}$. (right) The plot for the ratio of the first nonlinear quadratic friction term to the linear friction term for the $O_{1}$ constituent versus $\phi$ for the $M_{2}$ constituent.

\section{3-D Model Equations}

The primary difference between the 2-D and 3-D models is that in the 3-D formulation, nonlinear frictional effects can occur at the bottom by a quadratic slip condition (if used) as well as in the water column through a time variation in $E_{z}$. In sigma coordinates (setting $\sigma=0$ at the surface and $\sigma=-1$ at the bottom) the vertical shear stress in the water column has the form

$$
\frac{1}{H} \frac{\partial}{\partial \sigma}\left[\frac{\tau_{z}}{\rho_{0}}\right]=\frac{1}{H^{2}} \frac{\partial}{\partial \sigma}\left[E_{z} \frac{\partial u}{\partial \sigma}\right]
$$

while the quadratic slip bottom boundary condition is given by

$$
\frac{1}{H} \frac{\tau_{b}}{\rho_{0}}=\frac{1}{H} C_{f 3 \mathrm{D}}\left|u_{b}\right| u_{b}
$$

If we again expand the velocity and elevation about their time invariant parts, analytic expressions similar to (14) can be developed for (20) and (21)

$$
\begin{gathered}
\frac{1}{H} \frac{\partial}{\partial \sigma}\left(\frac{\tau_{z}}{\rho_{\mathrm{o}}}\right)_{n}=\tau_{z 3 D}+\left(\tau_{z 3 \mathrm{D}}^{\nu}+\ldots\right)+\left(\tau_{z 3 \mathrm{D}}^{\zeta}+\ldots\right) \\
\frac{1}{H}\left(\frac{\tau_{b}}{\rho_{\mathrm{o}}}\right)_{n}=\tau_{b 3 \mathrm{D}}+\left(\tau_{b 3 \mathrm{D}}^{\nu}+\ldots\right)+\left(\tau_{b 3 \mathrm{D}}^{\zeta}+\ldots\right)
\end{gathered}
$$

where

$$
\begin{gathered}
\tau_{b 3 D} \equiv \frac{C_{f 3 \mathrm{D}} \lambda_{b}}{h} u_{b n} \\
\tau_{b 3 \mathrm{D}}^{\nu} \equiv \frac{C_{f 3 \mathrm{D}}}{8 h \lambda_{b}} \underset{\substack{p, q, r \\
p \neq-q}}{\sum}\left(u_{b p} \cdot u_{b q}\right) u_{b r} \\
\tau_{b 3 \mathrm{D}}^{\zeta} \equiv-\frac{C_{f 3 \mathrm{D}} \lambda_{b}}{h^{2}} \sum_{p, q} \zeta_{p} u_{b q}
\end{gathered}
$$

In (22) and (23) the friction terms have again been separated into a linear component plus a nonlinear velocity and a nonlinear finite amplitude component. We note that (23) holds only for the quadratic slip bottom boundary condition (3-D cases 1 and 2). In these cases the slip condition results in a frictional effect that is identical to the 2-D case, except that the bottom slip velocity replaces the depth-averaged velocity in each expression. However, the frictional effect in the water column is different for each eddy viscosity formulation.

Case 1: $E_{z}=A_{1}|U| H$, quadratic slip bottom boundary condition. Using the case 1 eddy viscosity yields the following definition of terms in (22):

$$
\begin{gathered}
\tau_{z 3 \mathrm{D}} \equiv \frac{A_{1} \lambda}{h} \frac{\partial^{2} u_{n}}{\partial \sigma^{2}} \\
\tau_{z 3 \mathrm{D}}^{v} \equiv \frac{A_{1}}{8 h \lambda} \sum_{\substack{p, q, r \\
p \neq-q}}\left(U_{p} \cdot U_{q}\right) \frac{\partial^{2} u_{r}}{\partial \sigma^{2}} \\
\tau_{23 \mathrm{D}}^{\zeta} \equiv-\frac{A_{1} \lambda}{h^{2}} \sum_{p, q} \zeta_{p} \frac{\partial^{2} u_{q}}{\partial \sigma^{2}}
\end{gathered}
$$

An examination of (24) and (25) indicates that the ratios of the nonlinear to linear terms in both the bottom slip and the water column shear stress terms scale as the ratios in the 2-D model (i.e., $1 / 8 \lambda^{2}$ and $1 / h$ ). Therefore, for this choice of eddy viscosity, the calibration of the 2-D and 3-D models to produce essentially identical $M_{2}$ responses ensures that the overall model results will be similar, including those for the $\mathrm{M}_{4}$ and $\mathrm{M}_{6}$ overtides. Moreover, (24) and (25) imply that for the $|\boldsymbol{U}| \boldsymbol{H}$ eddy viscosity with a quadratic slip condition, the influence of the nonlinear friction terms on damping and overtide generation is the same regardless of whether the frictional dissipation occurs in the water column or at the bottom.

Case 2: $E_{z}=A_{2}|U|^{2}$, quadratic slip bottom boundary condition. Using the case 2 eddy viscosity yields the following definition of terms in (22):

$$
\begin{gathered}
\tau_{z 3 \mathrm{D}} \equiv \frac{A_{2} \lambda^{2}}{h^{2}} \frac{\partial^{2} u_{n}}{\partial \sigma^{2}} \\
\tau_{z 3 \mathrm{D}}^{\nu} \equiv \frac{A_{2}}{4 h^{2}} \sum_{\substack{p, q, r \\
p \neq-q}}\left(U_{p} \cdot U_{q}\right) \frac{\partial^{2} u_{r}}{\partial \sigma^{2}} \\
\tau_{z 3 \mathrm{D}}^{\zeta} \equiv-\frac{2 A_{2} \lambda^{2}}{h^{3}} \sum_{p, q} \zeta_{p} \frac{\partial^{2} u_{q}}{\partial \sigma^{2}}
\end{gathered}
$$


While the bottom slip condition retains the same form as in the 2-D model, the presence of $|\boldsymbol{U}|^{2}$ and the absence of a depth term in the eddy viscosity relation result in differences in the water column shear stress terms. In contrast with the 2-D model, the ratio of the nonlinear velocity term (26b) to the linear term (26a) scales with $1 / 4 \lambda^{2}$ and the ratio of the finite amplitude term (26c) to the linear term scales with $2 / h$. Therefore, in the water column, the nonlinear effects of friction are twice as large (relative to the linear term) with a $|\boldsymbol{U}|^{2}$ dependent eddy viscosity as they are with the 2-D model. As a result, for the same $M_{2}$ currents both the production of $\mathrm{M}_{4}$ and $\mathrm{M}_{6}$ overtides and the damping of secondary waves should be greater when the eddy viscosity is parameterized in terms of $|\boldsymbol{U}|^{2}$. Despite the fact that a majority of the friction is carried by the bottom slip term, this different nonlinear behavior is clearly observed in the Bight of Abaco runs (Figure 4).

In some studies [e.g., Davies and Aldridge, 1993] the $|\boldsymbol{U}| H$ and $|\boldsymbol{U}|^{2}$ dependent eddy viscosity formulations are used with a decreased eddy viscosity at the bottom and a larger slip coefficient. In effect, this shifts the bottom boundary condition closer to a no-slip condition and creates a more highly sheared velocity profile near the bed. However, the relationship between linear and nonlinear terms in the water column is unchanged. Assuming the depth-averaged $\mathbf{M}_{2}$ velocity is preserved, the results above suggest that such a change would produce similar $M_{4}$ and $M_{6}$ responses in the case of a $|\boldsymbol{U}| \mathrm{H}$ dependent viscosity but higher $\mathrm{M}_{4}$ and $\mathrm{M}_{6}$ responses in the case of a $|U|^{2}$ dependent viscosity (due to the increased velocity shear).

Case 3: $E_{z} \propto \kappa u_{*} z$, no slip bottom boundary condition. The shear velocity $u_{*}$ can be expanded in a harmonic series in a manner similar to (12). Using the case 3 eddy viscosity yields the following definition of terms in (22):

At $\sigma=-1$,

$$
\begin{gathered}
\tau_{z 3 \mathrm{D}} \equiv \frac{\kappa \lambda_{*} z_{0}}{h^{2}} \frac{\partial^{2} u_{n}}{\partial \sigma^{2}} \\
\tau_{z 3 \mathrm{D}}^{v} \equiv \frac{\kappa z_{0}}{8 h^{2} \lambda_{*}} \sum_{\substack{p, q, r \\
p \neq-q}}\left(u_{* p} \cdot u_{* q}\right) \frac{\partial^{2} u_{r}}{\partial \sigma^{2}} \\
\tau_{z 3 \mathrm{D}}^{\zeta} \equiv-\frac{2 \kappa \lambda_{*} z_{0}}{h^{3}} \sum_{p, q} \zeta_{p} \frac{\partial^{2} u_{q}}{\partial \sigma^{2}}
\end{gathered}
$$

For $-1<\sigma \leq-0.8$,

$$
\begin{aligned}
\tau_{z 3 \mathrm{D}} \equiv \frac{\kappa \lambda_{*}}{h}\left[\frac{\partial u_{n}}{\partial \sigma}+\left(\sigma+\frac{z_{0}}{h}\right) \frac{\partial^{2} u_{n}}{\partial \sigma^{2}}\right] \\
\tau_{z 3 \mathrm{D}}^{\nu} \equiv \frac{\kappa}{8 \lambda_{*} h}\left[\sum_{\substack{p, q, r \\
p \neq-q}}\left(u_{* p} \cdot u_{* q}\right) \frac{\partial u_{r}}{\partial \sigma}\right. \\
\left.+\left(\sigma+\frac{z_{0}}{h}\right)_{\substack{p, q, r \\
p \neq-q}}\left(u_{* p} \cdot u_{* q}\right) \frac{\partial^{2} u_{r}}{\partial \sigma^{2}}\right]
\end{aligned}
$$

$$
\begin{aligned}
\tau_{z 3 \mathrm{D}}^{\zeta} \equiv & -\frac{\kappa \lambda_{*}}{h^{2}}\left[\sum_{p, q} \zeta_{p} \frac{\partial u_{q}}{\partial \sigma}\right. \\
& \left.+\left(\sigma+\frac{2 z_{0}}{h}\right) \sum_{p, q} \zeta_{p} \frac{\partial^{2} u_{q}}{\partial \sigma^{2}}\right]
\end{aligned}
$$

For $-0.8 \leq \sigma \leq 0$,

$$
\begin{gathered}
\tau_{z 3 \mathrm{D}} \equiv \frac{0.2 \kappa \lambda_{*}}{h} \frac{\partial^{2} u_{n}}{\partial \sigma^{2}} \\
\tau_{z 3 \mathrm{D}}^{v} \equiv \frac{0.2 \kappa}{8 h \lambda_{*}} \sum_{\substack{p, q, r \\
p \neq-q}}\left(u_{* p} \cdot u_{* q}\right) \frac{\partial^{2} u_{r}}{\partial \sigma^{2}} \\
\tau_{z 3 \mathrm{D}}^{\zeta} \equiv-\frac{0.2 \kappa \lambda_{*}}{h^{2}} \sum_{p, q} \zeta_{p} \frac{\partial^{2} u_{q}}{\partial \sigma^{2}}
\end{gathered}
$$

where

$$
\lambda_{*}=\left(\frac{1}{4} \sum_{N} u_{*_{n}} \cdot u_{*_{n}}+u_{*_{0}} \cdot u_{*_{0}}\right)^{1 / 2}
$$

and the $u_{*_{n}}$ are complex harmonic amplitudes for the shear velocity.

In this case the relative behavior of the nonlinear terms varies with position in the water column. At the bottom the ratio of the nonlinear velocity term (27b) to the linear term scales with $1 / 8 \lambda_{*}^{2}$, and the ratio of the nonlinear finite amplitude term $(27 \mathrm{c})$ to the linear term scales with $2 / h$. Thus, at the bottom, the generation of the $M_{6}$ overtide will be similar to the 2-D model and the 3-D, case 1 model, while the generation of the $\mathrm{M}_{4}$ overtide will be similar to the 3-D, case 2 model and therefore will be enhanced over the 2-D model. In the upper $80 \%$ of the water column the two ratios scale with $1 / 8 \lambda^{2}$ and $1 / h$, respectively, suggesting a behavior similar to that found in the 2-D model. In the lower $20 \%$ of the water column, two characteristic behaviors are observed. Very close to the bottom, the ratio $z_{0} / h$ exceeds $\sigma$. Also, assuming a logarithmic velocity profile, $\partial^{2} u_{n} / \partial \sigma^{2}>\partial u_{n} / \partial \sigma$. Therefore close to the bottom, the ratios in (28) approach those at the bottom (27) and generation of the $\mathrm{M}_{4}$ overtide will be enhanced over the 2-D model. Away from the bottom, the value of $\sigma$ rapidly becomes $\gg z_{0} / h$ and the nonlinear velocity and finite amplitude ratios scale with $1 / 8 \lambda_{*}^{2}$ and $1 / h$, respectively, similar to the upper $80 \%$ of the water column. Model runs in the Bight of Abaco confirm that the $M_{4}$ generation is enhanced over the 2-D model, while the $M_{6}$ is not (Figure 5).

\section{Modified Friction Computations}

The results presented in sections 3 and 4 of this paper have shown that spatially variable friction, uncertainty in the bathymetric distribution, 2-D versus 3-D model formulations, and different eddy viscosity formulations are all unable to account for the consistent overprediction of the $M_{6}$ overtide. A possibility which has not been examined is that $M_{6}$ production in some areas could be reduced if element wetting and drying (not accounted for by the model) were allowed. However, if wetting and drying occurs at all, its influence 
would be limited to the shallowest areas very close to shore and therefore would not likely change the $M_{6}$ significantly in other regions. Since the predicted $M_{6}$ amplitudes are too high throughout the entire domain, we believe that allowing for element wetting and drying would have little effect on the overall model behavior.

Prior to pursuing the $M_{6}$ overprediction any further it is reasonable to consider the level of confidence we can have in these data and whether model overprediction is a result of bias in the observations. A review of the data reported by Filloux and Snyder [1979] suggests the observations are stable and well defined, and as shown in Table 1, observation errors are less than the prediction errors. Meteorological effects were taken into account in the original analysis by removing atmospheric pressures from the signals and by separating records into tidal and setup components. Data from stations occupied more than once are consistent despite differences in meteorological conditions during the three observation periods. Moreover, bias in model results exists only for the $M_{6}$ and not for the $M_{4}$. It seems likely that if $M_{6}$ observations were consistently low, $M_{4}$ observations (which are of the same order) would be low as well. Thus, bias in the measurements is probably not responsible for model overpredictions.

One remaining option is to modify the standard quadratic friction and eddy viscosity formulations. It has been demonstrated that nonlinear frictional interactions involving the $M_{2}$ constituent are responsible for the generation of the $M_{6}$ overtide and contribute to the damping of secondary astronomical constituents in both the 2-D and 3-D models. The principal difficulty in the Bight of Abaco is that while the $M_{2}$ is well represented in both models, the nonlinear interactions overgenerate the $M_{6}$ tide and may also overdamp the $\mathrm{O}_{1}, \mathrm{~K}_{1}$, and $\mathrm{S}_{2}$ tides. While the nonlinear frictional effects on the damping of the $M_{2}$ tide cannot be ignored, the response of the $\mathrm{M}_{2}$ is mostly due to the linear effects of friction. Therefore, if we decrease the nonlinear contribution relative to the linear contribution, we should be able to achieve proper damping of the $\mathrm{M}_{2}$ and, at the same time, reduce the production of the $\mathrm{M}_{6}$ overtide and the damping of the secondary astronomical constituents.

We investigate the impact of this idea in a final series of model runs using both the 2-D model and the 3-D, case 3 model. In both models we reduced the nonlinear part of the friction term by a fixed percentage while maintaining the linear part at approximately the same level. For the 2-D model this was accomplished by adding a linear contribution to the friction term, the magnitude of which was computed from the rms depth-averaged velocity. Thus the friction term in the 2$\mathrm{D}$ model becomes

$$
\frac{\tau_{b}}{\rho_{\mathrm{o}}}=C_{f 2 \mathrm{~d}}[(1-\beta)|\boldsymbol{U}|+\beta \lambda] U
$$

where $C_{f 2 \mathrm{~d}}=0.0085$ (the previous optimum value), $\beta$ is the nonlinear reduction factor (e.g., for a $30 \%$ nonlinear reduction, $\beta=0.3$ ), and $\lambda$ is the rms depth-averaged velocity computed from the original model run. For the 3-D model the reduction is applied to the eddy viscosity. For example, at the bottom the eddy viscosity becomes

$$
E_{z}=\left[(1-\beta) u_{*}+\beta \lambda_{*}\right] \kappa z_{0}
$$

where $\lambda_{*}$ is the rms shear velocity introduced following (29). The value $\lambda_{*}$ at each node was computed using a time series of the bottom stress from the original 3-D, case 3 run.

Typical results from this final set of runs are shown in Table 6. We considered a number of reduction percentages and found that a $30 \%$ reduction for the $2-\mathrm{D}$ model and a $20 \%$ reduction for the 3-D model provided the best overall results.

For the 2-D model the amplitude error for the $M_{6}$ is reduced from 35.5 to $21.1 \%$. In addition, the error distribution shows that the $\mathbf{M}_{6}$ amplitudes are no longer consistently overpredicted. Improvements in the overall error in the secondary astronomical constituents are less dramatic due to the reduced influence of the nonlinear terms on these constituents. Nevertheless, the error bias toward being overdamped has been largely eliminated for $O_{1}$ and $K_{1}$ and substantially reduced for $S_{2}$. Some improvement in the magnitude and distribution of the phase prediction errors is apparent as well. Overall, the results for the $M_{2}, N_{2}$, and $M_{4}$ are similar to the original model computations.

For the 3-D model the $\mathrm{M}_{6}$ amplitude error has been reduced by approximately one half and the bias in the error distribution has been nearly eliminated. The $\mathrm{M}_{4}$ amplitude error has been reduced, and the distribution slightly

\begin{tabular}{|c|c|c|c|c|c|c|c|}
\hline Measure & $O_{1}$ & $\mathbf{K}_{1}$ & $\mathbf{N}_{\mathbf{2}}$ & $\mathbf{M}_{2}$ & $\mathbf{S}_{\mathbf{2}}$ & $\mathbf{M}_{4}$ & $M_{6}$ \\
\hline \multicolumn{8}{|c|}{ 2-D Model Run* } \\
\hline Amplitude error, $\%$ & 4.9 & 8.0 & 21.7 & 7.9 & 18.2 & 20.6 & 21.1 \\
\hline Phase error, deg & 3.9 & 6.3 & 12.8 & 5.6 & 15.8 & 24.4 & 25.8 \\
\hline Amplitude overpredicted & 11 & 10 & 15 & 15 & 9 & 11 & 11 \\
\hline Amplitude underpredicted & 12 & 14 & 9 & 9 & 15 & 12 & 13 \\
\hline Phase overpredicted & 17 & 17 & 16 & 13 & 18 & 15 & 7 \\
\hline Phase underpredicted & 8 & 8 & 9 & 12 & 7 & 10 & 18 \\
\hline \multicolumn{8}{|c|}{ 3-D Model Runt } \\
\hline Amplitude error, $\%$ & 5.3 & 8.5 & 23.0 & 8.5 & 19.2 & 22.5 & 19.3 \\
\hline Phase error, deg & 4.2 & 6.0 & 12.8 & 5.9 & 16.1 & 25.0 & 22.7 \\
\hline Amplitude overpredicted & 17 & 15 & 15 & 15 & 7 & 13 & 10 \\
\hline Amplitude underpredicted & 6 & 9 & 9 & 9 & 16 & 9 & 13 \\
\hline Phase overpredicted & 14 & 18 & 18 & 14 & 18 & 14 & 11 \\
\hline Phase underpredicted & 11 & 7 & 7 & 11 & 7 & 11 & 14 \\
\hline
\end{tabular}

Table 6. Results for the 2-D and 3-D Models With Modified Friction Terms

- Model was run with a $30 \%$ nonlinear reduction.

$\dagger$ Model was run with a $20 \%$ nonlinear reduction. 
improved as well, although the change is small in comparison with the variability in the $\mathrm{M}_{4}$ observations. The overall amplitude errors for the $O_{1}, K_{1}$, and $N_{2}$ constituents are slightly higher in the present case, although the $O_{1}$ and $K_{1}$ have gone from being overdamped in the original 3-D run to being underdamped in this run.

One physical explanation for modifying the friction formulation is that the modifications account for the presence of a steady background current or residual turbulence field which contributes to the frictional processes. For example, the modified formulation for the 2-D model (30) may be rewritten

$$
\frac{\tau_{b}}{\rho_{\mathrm{o}}}=(1-\beta) C_{f 2 \mathrm{~d}}\left(|\boldsymbol{U}|+U_{\mathrm{o}}\right) U
$$

where $U_{0}$ is defined as $\beta \lambda /(1-\beta)$. Assuming $\beta=0.3$ and $\lambda \approx 25 \mathrm{~cm} / \mathrm{s}$ near the sill region, this corresponds to a steady background current of approximately $10 \mathrm{~cm} / \mathrm{s}$. Similarly, in the 3-D model, $\beta=0.2$ and $\lambda_{*} \approx 2.5 \mathrm{~cm} / \mathrm{s}$ corresponds to a background current of approximately $7 \mathrm{~cm} / \mathrm{s}$.

The assumption of steady background currents has been made in other modeling studies. In fact, S79 used an assumed steady current to reduce the $M_{6}$ response, although the required steady background velocity was considerably higher $(28 \mathrm{~cm} / \mathrm{s})$. However, their runs did not include the full complement of compound tidal interactions which are quite important in the Bight of Abaco. In addition, the effects on secondary astronomical constituents were not considered. The contribution of an assumed steady background current was included also by Naimie et al. [1994] in their model of circulation on Georges Bank. In their model the steady current was $7 \mathrm{~cm} / \mathrm{s}$.

A generic modification to the eddy viscosity in a 3-D model has also been proposed by R.A. Walters (manuscript in review, 1995) in order to improve the prediction of overdamped secondary astronomical constituents in the Delaware River. More accurate predictions of the $\mathrm{N}_{2}$ constituent were achieved by reducing the nonlinear contribution of the viscosity by $40 \%$.

\section{Conclusions}

In this paper we compare 2-D and 3-D models for computing the tidal response at five astronomical and two overtide frequencies in a shallow, friction-dominated embayment. The results indicate that although the 2-D and 3$D$ models may be calibrated to produce very similar responses for the dominant astronomical $\left(\mathrm{M}_{2}\right)$ tide, systematic differences exist in the computed responses of the overtides depending on the eddy viscosity formulation that is used.

Analytical expansions were developed to show that the differences in the 2-D and 3-D models are related to the relative influence of nonlinear velocity and finite amplitude components of the friction terms. For a 3-D model with a quadratic slip condition and a $|\boldsymbol{U}| H$ dependent eddy viscosity, the nonlinear terms behave almost identically with those in the 2-D model. Thus the computed amplitudes for all constituents are essentially identical in both models when they are calibrated to have similar $\mathbf{M}_{2}$ responses. In the case of a quadratic slip condition and a $U^{2}$ dependent viscosity the 3-D model produces consistently higher $M_{4}$ and $M_{6}$ amplitudes than the 2-D model due to the greater influence of the nonlinear terms associated with friction in the water column. When a no-slip condition and a $k u * z$ eddy viscosity are used, the $\mathrm{M}_{4}$ amplitudes are consistently higher in the 3$D$ model than in the 2-D model. For all the models the importance of the nonlinear terms is dependent on the ellipticity of the tidal currents; the strongest effects are found in rectilinear flow.

The results of this investigation indicate that the problems of overproduction of $\mathrm{M}_{2}$ overtides (in particular the $\mathrm{M}_{6}$ ) and overdamping of secondary astronomical tides often encountered in 2-D models will occur in 3-D models as well. These problems are shown to have a common origin, i.e., nonlinear frictional contributions that are too large relative to the linear frictional contribution. Although a number of causes were considered, it was possible to improve the overgeneration of the $M_{6}$ overtide and the overdamping of the secondary astronomical tides only by modifying the friction term to include an additional linear contribution. This additional contribution could represent a steady current, residual turbulence, or some other frictional process that is not properly accounted for by the standard analytical friction formulations considered in this study. If a steady current is assumed, a value of $7-10 \mathrm{~cm} / \mathrm{s}$ is required to meet the additional linear friction requirement.

If the analytical friction formulations are considered inadequate, a logical next stép might be to use a higher-order turbulence closure. In particular, the transient storage of turbulence in the water column may allow for the development of a residual turbulence field. However, in the shallow waters of the Bight of Abaco, turbulence production and dissipation will likely be in close balance throughout the water column, leaving little left over for a residual field to develop. Thus a higher-order turbulence closure is unlikely to overcome the difficulties encountered in the Bight of Abaco.

An interesting consequence of our analyses is an appreciation of the degree to which model formulations may be constrained by comparisons with elevation data for overtides and other nonlinear tides. Model runs in the bight showed that the overtides were much more sensitive than the astronomical tides to the choice of friction coefficient, 2-D versus 3-D formulation, eddy viscosity, and bathymetric depth.

Acknowledgments. Support for this work was provided by National Science Foundation grant OCE-9116448, Army Research Office contract P-30238-RT-AAS, and by U.S. Army Corps of Engineers Waterways Experiment Station Dredging Research Program contract DACW39-90-K-0021. This work benefited from discussions with Roy Walters and reviews by A.M. Davies and one additional reviewer.

\section{References}

Aldridge, J.N., and A.M. Davies, A high resolution three dimensional hydrodynamic tidal model of the eastern Irish Sea, J. Phys. Oceanogr., 23, 207-224, 1993.

Bowden, K.F., L.A. Fairbum, and P. Hughes, The distribution of shearing stresses in a tidal current, Geophys. J. R. Astron. Soc., 2, 288-305, 1959.

Bowers, D.G., T.P. Rippeth, and J.H. Simpson, Tidal friction in a sea with two equal semidiurnal constituents, Cont. Shelf Res., 11 , 203209, 1991.

Davies, A.M., A bottom boundary layer resolving three-dimensional tidal model: A sensitivity study of eddy viscosity formulations, $J$. Phys. Oceanogr., 23, 1437-1453, 1993 a.

Davies, A.M., Numerical problems in simulating tidal flows with a 
frictional-velocity-dependent eddy viscosity and the influence of stratification, Int. J. Numer. Methods Fluids, 16, 105-131, 1993b.

Davies, A.M., and J.N. Aldridge, A numerical model study of parameters influencing tidal currents in the Irish Sea, J. Geophys. Res., 98, 7049-7067, 1993.

Davies, A.M., and G.F. Furnes, Observed and computed $M_{2}$ tidal currents in the North Sea, J. Phys. Oceanogr., 10, 237-257, 1980.

Filloux, J.H., and R.L. Snyder, A study of tides, setup and bottom friction in a shallow semi-enclosed basin, I, Field experiment and harmonic analysis, J. of Phys. Oceanogr., 9, 158-169, 1979.

Godin, G., Frictional effects in river tides, in Tidal Hydrodynamics, edited by B.B. Parker, pp. 379-402, John Wiley, New York, 1991.

Kinnmark, I., The Shallow Water Wave Equations: Formulation Analysis and Application, Lect. Notes in Eng., vol. 15, edited by C.A. Brebbia and S.A. Orszag, 187pp., Springer Verlag, New York, 1985.

Kolar, R.L., J.J. Westerink, and M.E. Cantekin, Aspects of nonlincar simulations using shallow water models based on the wave continuity equations, Comput. Fluids, 23, 523-538, 1994.

LeProvost, C., Generation of overtides and compound tides (revicw), in Tidal Hydrodynamics, edited by B.B. Parker, pp. 269-295, John Wiley, New York, 1991.

LeProvost, C., and M. Fornerino, Tidal spectroscopy of the English Channel with a numerical model, J. Phys. Oceanogr., 15, 1009-1031, 1985.

Luettich, R.A., Jr., and J.J. Westerink, A solution for the vertical variation of stress, rather than velocity, in a three-dimensional circulation model, Int. J. Numer. Methods Fluids, 12, 911-928, 1991.

Luettich, R.A., Jr., J.J. Westerink, and N.W. Scheffner, ADCIRC: An Advanced Three-Dimensional Circulation Model for shelves, coasts and estuarics, I, Theory and methodology of ADCIRC-2DDI and ADCIRC-3DL, DRP Tech. Rep. I, 137 pp., U.S. Army Corps of Eng. Waterways Exp. Stn., Vicksburg, Miss., 1992.

Luettich, R.A., Jr., S. Hu, and J.J. Westerink, Development of the direct stress solution technique for threc-dimensional hydrodynamic models using finite elements, Int. J. Numer. Methods Fluids, 19, 295-319, 1994.

Lynch, D.R., and W.G. Gray, A wave equation model for finite element tidal computations, Comput. Fluids, 7, 207-228, 1979.

Lynch, D. R., and C.E. Naimie, The $M_{2}$ tide and its residual on the outer banks of the Gulf of Maine, J. Phys. Oceanogr., 23, 2222$2253,1993$.

Naimie, C.E., J.W. Loder, and D.R. Lynch, Scasonal variation of the three-dimensional residual circulation on Georges Bank, J. Geophys. Res., 99, 15,967-15,989, 1994.

Parker, B.B., The relative importance of the various nonlinear mechanisms in a wide range of tidal interactions (review), in Tidal Hydrodynamics, edited by B.B. Parker, pp. 237-268, John Wiley, New York, 1991.
Pingree, R.D., Spring tides and quadratic friction. Deep Sea Res., Part $A, 30,929-944,1983$.

Pingree, R.D., and D.K. Griffiths, Tidal friction for semi-diurnal tides, Cont. Shelf Res., 7, 1181-1209, 1987.

Proudman, J., Dynamical Oceanography, 409pp., Dover, Mineola, N.Y., 1953.

Sidjabat, M.M., The numerical modeling of tides in a shallow, semienclosed basin by a modified elliptic method, Ph.D. thesis, 96 pp., Univ. of Miami, Coral Gables, Fla., 1970.

Snyder, R.L., M. Sidjabat, and J.H. Filloux, A study of tides, setup and bottom friction in a shallow semi-enclosed basin. II, Tidal model and comparison with data, J. Phys. Oceanogr, 9, 170-188, 1979.

Soulsby, R.L., Tidal-current boundary layers, in The Sea, edited by B. LeMehaute and D.M. Hades, pp. 523-566, John Wiley, New York, 1990.

Speer, P.E., and D.G. Aubrey, A study of non-linear tidal propagation in shallow inlet/estuarine systems, II, Theory, Estuarine Coastal Shelf Sci., 21, 207-224, 1985.

Sucsy, P.V., B.R. Pearce, and V.G. Panchang, Comparison of two- and three-dimensional model simulation of the effect of a tidal barrier on the Gulf of Maine tides, J. Phys. Oceanogr., 23, 1231-1248, 1993.

Walters, R.A., A finite element model for tidal and residual circulation, Commun. Appl. Numer. Methods, 2, 393-398, 1986.

Walters, R.A., A model for tides and currents in the English Channel and southern North Sea, Adv. Water Resour., 10, 138-148, 1987.

Walters, R.A., A three-dimensional, finite element model for coastal and estuarine circulation, Cont. Shelf Res., 12, 83-102, 1992.

Walters, R.A., and F.E. Werner, Nonlinear generation of overtides, compound tides, and residuals, in Tidal Hydrodynamics, edited by B.B. Parker, pp. 296-320, John Wiley, New York, 1991.

Westerink, J.J., K.D. Stolzenbach, and J.J. Connor, General spectral computations of the nonlinear shallow water tidal interactions within the Bight of Abaco, J. Phys. Oceanogr., 19, 1348-1371, 1989.

Westerink, J.J., R.A. Luettich Jr., and J.C. Muccino, Modeling tides in the western North Atlantic using unstructured graded grids, Tellus Ser. $A, 46,178-199,1994$.

R.R. Grenier Jr., Department of Civil Engineering, North Carolina State University, Raleigh, NC 27695.

R.A. Luettich Jr., Institute of Marine Sciences, University of North Carolina at Chapel Hill, 3431 Arendell Street, Morehead City, NC 28557. (e-mail: Rick_Luettich@unc.edu)

J.J. Westerink, Department of Civil Engineering and Gcological Sciences, University of Notre Dame, Notre Dame, IN 46556.

(Reccived October 24, 1994; revised February 6, 1995; accepted March 10, 1995.) 